\title{
Choles y Chortíes de Karl Sapper, 1907
}

\author{
Editores: Axel Michael Köhler y Víctor Manuel Esponda Jimeno
}

\section{Presentación}

$\mathrm{E}$ 1 documento que presentamos casi alcanza la centuria de haberse publicado y ésta es la primera versión castellana que se hace de él; hay una traducción al inglés de escasa circulación que es la que más se cita, mas el texto alemán original apenas si se conoce; asimismo la publicación en que vio la luz primera no es del todo accesible.

Es uno de los primeros trabajos de síntesis sólidos en torno a la historia, etnografía y lingüística de los grupos cholanos de Chiapas y Guatemala. En él se presentan diversas ideas referentes a la lengua que hablaron los extintos lacandones, a la vez que se presentan datos históricos y lingüísticos que indican que el chol fue la lengua que hablaron dichos lacandones y que los actuales homónimos son de origen maya peninsular.

El trabajo de Sapper retoma datos importantes que antiguos historiadores expusieron acerca de la historia y lengua de los grupos choltí, poconchí, chortí, pokomán y chol, y con esa información y sus propias pesquisas llevadas a cabo por Chiapas y Guatemala nos da a conocer sus valiosas impresiones acerca de un asunto que modernos historiadores creyeron haber descubierto:

Axel Michael Köhler y Víctor Manuel Esponda Jimeno

CESMECA-UNICACH
Sapper y los cronistas antiguos lo habían puesto de manifiesto muchísimo tiempo atrás.

La validez de este documento -desconocido para la generalidad de los habitantes donde tuvo su origen-es incuestionable y dará importantes pistas a los estudiosos de la lingüística maya; no obstante, debe advertirse que en el apartado de los vocabularios hay deficiencias notables de interpretación y registro que los lingüistas sabrán enmendar o discutir. Dichos vocabularios no se encuentran transcritos en el alfabeto fonético internacional en uso ahora y están presentados de la manera en que Sapper los escuchó utilizando para su transcripción, como nos informa en la página 440 del trabajo original, "la ortografía [...] española con las modificaciones propuestas por Otto Stoll (Etnografia, p. 39 ss.)”.

La publicación en castellano de este trabajo pionero enriquece la escasa literatura de la etnia chol, etnia que, dicho sea de paso, ha sido considerada por algunos estudiosos como la constructora de las grandes ciudades mayas que hay dispersas en la selva chiapaneca y guatemalteca.

A la presente traducción se le agregaron algunas notas de los editores para aclarar puntos imprecisos o para proporcionar información adicional. 


\section{E1 autor}

Karl Theodor Sapper nació el 6 de febrero de 1866 en Wittislingen, un pequeño pueblo alemán cercano a la frontera regional entre Bavaria y Suavia. De 1884 a 1888 estudió ciencias naturales en Munich terminando con una tesis geológica para obtener el doctorado. El mismo año de su graduación, en 1888, pasó a Guatemala donde se dedicó por un tiempo (aproximadamente dos años) a administrar la finca cauchera y cafetalera de su hermano Richard Sapper, el entonces vicecónsul del emperador alemán (kaiserlich Deutscher vicecónsul) en Cobán. Además de dicho trabajo, Karl Sapper se dio a la tarea de estudiar la geología, topografía, botánica y etnología de Cobán; a él se deben también los primeros mapas modernos de la geología y topografía de Guatemala, el Sureste de México y Belice, con base científica (publicados en "Über Gebirgsbau und Boden des nördlichen Mittelamerika", Petermanns Geographische Mitteilungen, Ergänzungsheft núm. 127, Gotha: Justus Perthes, 1899 [“'Sobre las estructuras montañosas y los suelos del Norte de Mesoamérica", Los Comunicados Geográficos de Petermann, suplemento núm. 127]). Sapper se quedó en América Central hasta 1900, donde efectuó cada año viajes importantes, base de sus investigaciones. En 1889 se fue de Cobán al noroeste de Guatemala y sureste de Chiapas, en 1890 al lago Izabal y las ruinas de Quiriguá, en 1891 al sur del Petén, a Yaxchilán y Polochic, en 1892 a las Honduras Británicas, etcétera. Entre 1893 y 1894 trabajó en el sureste de México -el Istmo de Tehuantepec, Chiapas, Tabasco y Yucatán- en calidad de geólogo en servicios mexicanos. En 1895 regresó a Alemania por breve tiempo, pero en 1896 volvió a América Central donde continuó sus investigaciones en Honduras, El Salvador, Nicaragua, Panamá y Costa Rica. Los resultados publicados de dichos trabajos fundaron su reputación como uno de los más experimentados y acuciosos geógrafos y geólogos modernos de la región.
Regresó a su patria en 1900 habilitándose en Leipzig con Friedrich Ratzel como "Privatdozent" [grado académico en Alemania que es requisito para obtener una plaza como profesor catedrático], y en 1902 obtuvo la cátedra de Geografía en Tübingen. En el verano de 1902 hasta 1903 viajó de nuevo a las Américas, esta vez pasando por los Estados Unidos y México a América Central, con objeto de estudiar los fenómenos volcánicos de esa región y de las Antillas. Los viajes e investigaciones del resto de esta década fueron igualmente dedicados a los estudios vulcanológicos. Fue nombrado en 1908 profesor catedrático y director del Seminario Geográfico de la Universidad de Tübingen, se desempeñó además como catedrático en las universidades de Estrasburgo entre 1910 y 1918 y de Würzburgo desde 1919, donde trabajó hasta jubilarse en 1932. En 1923 fundó aquí el "Institut für Amerikaforschung an der Universität W ürzburg" [Instituto de Estudios Americanistas de la Universidad de Würzburgo].

Sus últimos días los pasó en el pueblo de Garmisch en los Alpes de Bavaria, donde falleció el 29 de marzo de 1945.

\section{La obra}

La bibliografía de la obra completa de Sapper se encuentra en la biografía escrita por su alumno Franz Termer: Karl Theodor Sapper. 1866-1945. Leben und Wirken eines deutschen Geographen und Geologen. Leipzig: Johann Ambrosius Barth, 1966.

Dentro de sus más de 450 publicaciones en alemán cabe mencionar las más importantes respecto a la región centroamericana:

- "Grundriss der physikalischen Geographie von Guatemala." Petermanns Geographische Mitteilungen, Ergänzungsheft No. 113, 59 pp. con 4 mapas. Gotha: Justus Perthes, 1894 [“'Sobre las estructuras 
montañosas y los suelos del Norte de Mesoamérica", Los Comunicados Geográficos de Petermann, suplemento núm. 113]);

- Das nördliche Mittelamerika nebst einem Ausflug nach dem Hochlandvon Anahuac. Reisen und Studien aus den Jabren 1888-1895. Braunschweig: Vieweg \& Sohn, 436 pp. con 8 mapas, 1897 [El norte de Mesoamérica y una excursión a los Altos de Anabuac. Viejesy estudios de los años 1888 hasta 1895];

- "Über Gebirgsbau und Boden des nördlichen Mittelamerika". Petermanns Geographische Mitteilungen, Ergänzungsheft No. 127, 119 pp. con 3 mapas y 25 perfiles geológicos. Gotha: Justus Perthes, 1899 ["Sobre las estructuras montañosas y los suelos del Norte de Mesoamérica”];

- "Die Alta Verapaz (Guatemala), eine landeskundliche Skizze". Mitteilungen der geographischen Gesellschaft, No. 17, pp. 78-224 con 5 mapas. Hamburg, 1901 [“Alta Verapaz (Guatemala), un esbozo topográfico”. Los Comunicados de la Sociedad Geográfica];

- Mittelamerikanische Reisen und Studien aus den Jabren 1888 bis 1900. Braunschweig: Friedrich Vieweg \& Sohn, 426 pp. con 60 figuras y 4 mapas, 1902 [Viajes y estudios mesoamericanos de los años 1888 hasta 1900];

- "Die Zukunft der mittelamerikanischen Indianerstämme". Archiv der Rassen- und Gesellschaftsbiologie, No. 2, pp. 383-413, 1905. [“El futuro de las étnias indígenas de Mesoamérica". Archivo de la Biología Racial y Social]

- "Über Gebirgsbau und Boden des südlichen Mittelamerikas". Petermanns Geographische Mitteilungen, Ergänzungsheft No. 151, 82 pp. con 2 mapas y 2 perfiles geológicos. Gotha: Justus Perthes, 1905
[“Sobre las estructuras montañosas y los suelos del sur de Mesoamérica"];

- In den Vulkangebieten Mittelamerikas und Westindiens. Reiseschilderungen und Studien über die Vulkanausbrüche der Jahre 1902 bis 1903, ibre geologischen, wirtschaflichen und sozialen Folgen. Stuttgart: Schweizerbart, 334 pp. con 76 figuras, 1905; [En las regiones volcánicas de Mesoamérica y el oeste de la India. Observaciones viajerasy estudios sobre las erupciones volcánicas de los años 1902 y 1903 incluyendo sus efectos geológicos, económicos y sociales.]; - Wirtschaftsgeographie von Mexiko. Halle/Saale: GebauerSchwetschke; Reihe III, Band 5 von Angewandte Geographie, herausgegeben von Hugo Grothe; 115 pp. con 6 diagramas, 1908 [Geografía económica de México. Serie III, Vol. 5 de Geografía Aplicada, edición de Hugo Grothe];

- "Über einige Sprachen von Südchiapas". En: Reseña de la $2^{a}$ sesión del XVII Congreso Internacional de Americanistas efectuada en la ciudad de México durante el mes de septiembre del 1910 (Congreso del Centenario), México, 1912, pp. 295-320 [“Sobre algunas lenguas del Sur de Chiapas'];

- "Die mittelamerikanischen Vulkane". Petermanns Geographische Mitteilungen, Ergänzungsheft No. 178, 173 pp. con 5 figuras y 1 mapa. Gotha: Justus Perthes, 1913 [“Los volcanes de Mesoamérica”];

- "Mittelamerika". Auslandswegweiser, No. 5, 124 pp. Hamburg: Friedrichsen u. Co., 1921. ["Mesoamérica"]

- Über den Charakter und die geistige Veranlagung der Kekchí Indianer". In: Festschrift Eduard Seler, pp. 401-440. Stuttgart, 1922 [“Sobre el carácter y la disposición mental de los indígenas q’eqchi'es”. En: Homenaje a Eduard Seler] 
- Mexiko. Land, Volk und Wirtschaft. 2. vollständig neubearbeitete Auflage der Wirtschaftsgeographie von Mexiko. Wien: Seidel und Sohn; 164 pp. con 28 figuras, 1928 [México. Elpaís, su gente y su economía. 2a edición, completamente revisada de Geografía económica de México];

- "Reise nach Süd und Mittelamerika, 1927/28". Mitteilungen der Geographischen Gesellschaft, zu Würzburg, III./IV.Jahrgang, pp. 1-177. Würzburg: E. Mönnich, 1929 ["Viaje al Cono Sur y Mesoamérica"];

- "Die Besitzergreifung Amerikas durch die Indianer". Ibero-Amerikanisches Archiv, No. 7, pp. 350-368, 1933 ["La colonización de las Américas por los indios". Archivo Iberomericano];

- "Der Kulturzustand der Indianer vor der Berührung mit den Europäern und in der Gegenwart". Verhandlungen des 24. Internationalen Amerikanisten-Kongresses in Hamburg 1930, pp. 73-96, 1934 ["El estado cultural de los indios antes del contacto con los europeos y en el presente". Memoria del xxiv Congreso Internacional de Americanistas celebrado en Hamburgo en 1930]

- "Die Verapaz im 16. und 17. Jahrhundert. Ein Beitrag zur historischen Geographie und Ethnographie des nordöstlichen Guatemala". Abhandlungen der Bayerischen Akademie der Wissenschaften, Mathematisch-Naturwissenschaftliche Abteilung; Neue Folge, Heft 37, 46 pp. con 2 mapas. München: C.H. Beck, 1936. ['La Verapaz en los siglos Xvi y XVII. Una contribución a la geografía y etnografía del noreste de Guatemala". Tratados de la Academia Bávara de las Ciencias, Departamento de las Ciencias Matemáticas y Naturales; Nueva Serie, Cuaderno 37];
- "Beiträge zur Besitzergreifung Amerikas und zur Entwicklung der altamerikanischen Landwirtschaft durch die Indianer". Mitteilungen des Museums für Völkerkunde Hamburg, No. 19, 44 pp. con 18 figuras, 1938 ["Contribuciones a la colonización de las Américas por los indios y el desarrollo de la agricultura americana antigua". Los Comunicados del Museo Etnológico de Hamburgo].

Hay un número limitado de las publicaciones de Sapper en castellano y pocas traducciones de su obra a esta lengua; respecto a la región centroamericana destacan las siguientes:

- "Informe sobre la geografía física y la geología de Chiapas y Tabasco". Boletin de Agricultura, Minería e Industrias No. 3, pp. 187-211. México, 1894.

- "Sobre la geografía física y la geología de la Península de Yucatán”. Boletín del Instituto Geológico, No. 3, 57 pp. con 4 mapas. México, 1896.

- Sobre la geografía física, la población y la producción de la República de Guatemala. Guatemala, 1897.

- Los volcanes de la América Central. Halle/Saale: Niemeyer, Estudios sobre América y España, Serie Extra 1, 116 pp. con 5 figuras, 1925.

- "La lengua tapachulteca". ElMéxico Antiguo, No. 2, pp. 259-268, México, 1927.

- "La población autóctona de la América Central". An. Soc. Argentina Estud. Geogr. "Gaea”, pp. 5-15, 1928.

- "Un viaje al nuevo mundo". Traducido del alemán por Evelin Paap. Mesoamérica, Año 2, Cuaderno 2, pp. 153-169, 1981. 
- "Alimentos y bebidas de los q'eqchi'es"; "Fray Bartolomé de las Casas y la Verapaz"; "Las costumbres y creencias religiosas de los q'eqchi'es." En: Karl Sapper, Eduard Seler and Franz Termer Estudios Q'eqchi'es: Etnógrafos Alemanes en las Verapaces. Traducido del alemán por Fernando Peñalosa. Cleveland, Ohio: Yax Te’ Books, 1998.
- Las ruinas de Bolonchac cercanas a San Bartolomé de los Llanos, 1893, apéndice de "Santo Ton, una fortaleza del Postclásico Temprano”, pp. 247-252, Víctor Manuel Esponda J. 1996, en Quinto Foro de Arqueología de Chiapas, 1996:233-252, Tuxtla Gutiérrez, Chiapas. 


\title{
Choles y Chortíes*
}

\author{
Karl Sapper
}

E

1 Dr. Otto Stoll, en el mapa adjunto de su trabajo etnográfico fundamental ${ }^{1}$ sobre Guatemala, asignó a los choles un amplio territorio que va desde el Noreste de Chiapas, pasando por el Petén y el lago de Yzabal, hasta el Golfo de Amatique. Para este trabajo, Stoll se basó, principalmente, en los referencias del Isagoge Histórico Apologético General de todas las Indiasy Especial de la Provincia de Chiapasy Guatemala 2 . En cuanto a los chortíes los ubicó únicamente la parte delEste de Guatemala que se encuentra al Sur del territorio chol. Stoll, refiriéndose al material recabado por el Dr. Berendt, identificó que la lengua de los choles era pariente cercana del chontal y de la lengua maya. El chortí, sin embargo, lo supuso como pariente cercano del pokomam, basándose en el vocabulario escasísimo que recopiló Stephens ${ }^{3}$. En los diferentes viajes que hice a estas regiones ${ }^{4}$ he podido darme cuenta de que la lengua chol se habla en la actualidad sólo en la parte Noreste de Chiapas y en los lugares colindantes de Tabasco (Xicotencal), mientras que la lengua chortí sigue viva en la parte Este del departamento guatemalteco de Chiquimula y lugares colindantes de la República de Honduras. He averiguado además que el vocabulario recopilado por Stephens no pertenece al chortí, sino al pokomam. Presumiblemente Stephens se equivocó

Karl Sapper, Universidad de Tübingen. entrevistando a un indígena que iba de paso por estas tierras. Como lo voy a demostrar a través de los vocabularios anexos, la verdadera lengua chortí se presenta en la actualidad tan cercana del chol que la podemos clasificar más bien como un dialecto del chol y no como lengua aparte. Por ello también la mayoría de los autores antiguos han considerado a los chortíes como parte integral de los choles.

La distribución y afiliación actuales de las lenguas chol y chortí están comprobadas entonces de manera satisfactoria. Queda la pregunta de cómo estaba en el pasado.

En su informe al Rey de España en el año $1576^{5}$, Diego García de Palacio señala que la lengua de Chiquimula de la Sierra y de los alrededores de Copán es el apay, y agrega que "según antiguas tradiciones orales hace mucho tiempo que un pueblo de Yucatán conquistó y sometió a las provincias de Ayajál, Lacandón, Verapaz, así como a las regiones de Chiquimula y de Copán. Además la lengua apay se usa y se entiende en Yucatán y en otras provincias." (De hecho, como subraya Stoll [op. cit., p. 91], Marina, la traductora de Cortez, pudo efectivamente comunicarse con los habitantes dela región del lago Yzabal). Para Verapaz, García de Palacio señala el poconchí y el caechicolchí, y es claro que se trata de las lenguas poconchí y kekchí que se hablan todavía, así que 
una tercera lengua, el “colch?”, que podemos considerar sinónimo de apay. La palabra "cholchî" parece ser un error y es probable que se trata de "la lengua chol", expresión que los indígenas kekchí siguen usando. Lo que llama la atención es que en los tiempos de García de Palacio aparentemente ya se había dado el cambio fonético de ' $\mathrm{t}$ ' a 'ch' en la región de Verapaz, mientras que en el año 1695, fray Francisco Morán ${ }^{6}$ todavía u tilizaba la palabra "choltr" para señalar a la lengua de la Villa de Dolores. Desgraciadamente no he podido tener acceso al vocabulario de Morán y esto es todavía más lamentable dado que deberíamos suponer -según las informaciones históricas que tenemos-que el vocabulario en cuestión fue transcrito inmediatamente después de la conquista del Lacandón (Nuestra Señora de los Dolores). En esta obra la lengua de los lacandones está expresamente designada como el "choltî", eso quiere decir que para esta época podemos afirmar que el chol se hablaba en el Este de Chiapas hasta el río Usumacinta. Lapequeña muestra lingüística que nos ofrece Villagutierre y Sotomayor ${ }^{7}$ (p. 262): “ut: impusidal", ${ }^{8}$ no nos lo indica con toda certeza, porque en la lengua chol actual la frase “mi corazón está bien" debe expresarse como "utz ni pusical'. Pero parece que en las lenguas indígenas a veces se cambia el $n i$ en im -por ejemplo, según el Comunicado en Cakchiquel (Mitteilung im Cakchiquel) de O. Stoll-entonces es muy probable que estamos frente al verdadero chol. Sin embargo, el hecho que la lengua de los lacandones esté designada "chaltî", no nos ofrece una prueba absoluta de que se trata realmente del chol, porque los choles mismos llaman a su lengua "putum". Y si tomamos como referencia el uso lingüístico actual de los propios indígenas kekchí, debemos connotar el nombre de "lengua chol" únicamente como una lengua bárbara, porque los indígenas kekchí entienden por "chol cvüink" a todos los indígenas paganos de sus alrededores, sobre todo los lacandones hablantes de maya del Petén. Las lenguas de San Luis en el Petén y de San Antonio en las Honduras Británicas que los kekchí expresamente designan como "cholchí", son dialectos mayas que se distinguen poco. ${ }^{9}$ Bajo tales circunstancias, tampoco resulta ser prueba irrefutable para la existencia anterior de la lengua chol en el norte de Alta Verapaz, lo que nos dice expresamente el Padre Alonso de Escobar. ${ }^{10}$ Según él, los descendientes de los lacandones que los dominicos habían sometido y reducido a finales del siglo XvI de Chamá al barrio de San Marcos (Cobán), todavía en su época, es decir en la primera mitad del siglo xIx, hablaban entre ellos la lengua echolchi, "wbich is that of the L acandones" [que es la de los lacandones. N. de los E.]. Agrega que, "The division of San Tomas Apostol is as ancient as the Conquest, and was peopled with Lacandon Indians dwelling to the North of Coban. In like manner San Domingo de Coban was established with Indians taken from the mountains of Chichen and Xucamel.11 The four divisions of San Pedro Carcha were peopled with the Indians of the immediate neigbourbood. In general the Indian communities of S an Pedro and Coban still gather the produce of those tracts of country which anciently belonged to their respective ancestors"*

La región de Chamá estaba originalmente habitada por indígenas poconchí, según se deduce de un manuscrito poconchí que se conserva en San Cristóbal Verapaz —elllamado "Título del Barrio de Santa Ana"12 cuyo original fue escrito en 1565 y que desde luego fue evidentemente copiado varias veces con diversas adiciones- que indígenas poconchí de Chamá se trasladaron a San Cristóbal. Más tarde la gente de San Marcos reclamaron la región de Chamá, pero el título de Santa Ana (en la traducción de Vicente A. Narciso) dice de manera expresa, aparentemente en una adición que data de inicios del siglo XVII: "no son (los cerros y planos en Chamjah y Chichun) de los de San Marcos, porque muy lejos quedaron ellos en Chixa en Acalá, porque son de Acalá los de San Marcos, donde murió nuestro señor Santo Padre Fray Domingo de Vico siendo todavía los abuelos de los Padres de los de San Marcos, que mataron y se comieron al Padre Fray Domingo de Vico los de Acalá; el siguiente Padre Fray Alonso de 
Bayllo trajó a los de San Marcos; primero vivieron en Yax Capnal", ${ }^{13}$ salieron de allí y vivieron en seguida en Akil, salieron de allí de Akil; cuando hicieron esta salida pasaron al otro lado del río Chamjah a ocupar nuestros cerros y nuestros planos de nosotros los de Santa Ana". Parece que el reclamo de los poconchí no tuvo éxito, pues si de veras la gente de San Marcos fue reubicada a Cobán solamente pudo ser en el siglo XVIII. Sin embargo, no hay ninguna duda de que hubo una reubicación de Chamá a Cobán, porque en Chamá hay una leyenda sobre tal acontecimiento y además existe el apellido local Chamam en el barrio de San Marcos, lo que nos da una pista acertada. Según las indicaciones que encontramos en el título poconchí podemos suponer que el asentamiento original de la gente de San Marcos se ubicaba aproximadamente en los alrededores de la región de las Salinas de los Nueve Cerros, porque Yax Cabnal también se encuentra en el camino por allá. Además los acalanes paganos tenían como aliados a los lacandones ${ }^{14}$ en el ataque contra el pueblo cristiano de Acalá, donde fue asesinado fray Domingo de Vico. Los lacandones vivieron en ambos lados del río Chixoy y en la región Oeste del río, esto, si tomamos en cuenta las indicaciones poco claras de los autores antiguos. Podemos pensar entonces que la zona Acalá se ubicaba principalmente entre el río Chixoy y el río de la Pasión. Sabemos también por el informe de Cortez al emperador Carlos V que la zona Acalá incluía una región ubicada al Este del río Usumacinta y mucho más al Norte, y a ésta se refiere probablemente el comunicado de Villagutierre ${ }^{15}$ que relata la conquista de dicha zona desde Yucatán en la primera mitad del siglo XVI.

Por el momento y con todo lo que hemos comentado, debemos advertir que carecemos de una prueba precisa de que la lengua chol es la de los lacandones y acalanes, aunque la designación de estos dos idiomas como choltí y echolchí lo hace muy probable. Los pocos topónimos de localidades del Norte de Alta Verapaz que seguramente fueron traducidos ${ }^{16}$ no son tan importantes para esta cuestión porque pueden referirse tanto al chol como al maya. Las indicaciones arqueológicas tampoco nos dan una certeza absoluta acerca de este asunto. Se ha encontrado, por ejemplo, mucho menos obsidiana - en forma de puntas de flecha, puntas de lanza o de cuchillos - al Norte de las montañas Pocolhá que al Sur; en este punto dicho material ha sido remplazado por el sílex que se encuentra en el Petén. Esto solamente nos muestra que al Norte de dichas montañas vivían etnias que debían haber llegado del norte, pero no podemos saber si estas etnias fueran choles o mayas.

Es cierto que los lacandones que hoy día viven en el Petén y en el Este de Chiapas hablan maya, pero con esto no se puede comprobar que los antiguos lacandones también hablaran la misma lengua, porque el conocimiento de los españoles acerca de los lacandones siempre fue muy limitado y el nombre que le dieron al grupo étnico que encontraron probablemente se refería a la localidad y no a las particularidades etnográficas o lingüísticas de la gente. Seler, ${ }^{17}$ sin embargo, se inclina a pensar que los lacandones de los siglos XVI y XVII asentados en el este de Chiapas también hablaban maya. Dado que los textos transmitidos indican que pertenencia al chol, considero muy probable que su lengua fue el chol, aunque me reservo el beneficio de la duda.

Lo que es cierto es que en el siglo XVII vivieron choles en el noreste de Alta Verapaz, aunque parece que ya en aquellos tiempos eran muy pocos, según se deduce de todos los comunicados que tenemos a nuestra disposición. Lo mismo se puede decir respecto de los lacandones que insistentemente algunos autores han pretendido encontrar en vano en las vastas selvas vírgenes del Este de Chiapas. Según estimaciones hechas en 1676, ${ }^{18}$ los choles en el Norte y Este de Verapaz sumaban por lo menos 30000 personas. Lamentablemente, muy pocos de los topónimos que se encuentran en diversas obras históricas pueden ser identificados. También ignoramos la ubicación de la frontera entre los curatos 
de Verapaz y los de Castillo (San Felipe del lago Yzabal). Es probable que la frontera estuviera en el río Maytol o Factun, lo que corresponde a Sarstoon que los indígenas llaman Sactun (por lo tanto Factun sería una errata, en vez de Sactun).

En la región al Noreste de Verapaz en 1675, los frailes Gallegos y Delgado ya habían reducido una cantidad de choles en tres aldeas (San Lucas, El Rosario y Santiago). $\mathrm{El}$ año siguiente ambos regresaron con los choles, manchés y axoyes, "que todos vienen a ser uno, aunque de distintas parcialidades," bautizaron y concentraron una gran cantidad de gente en aldeas, así que ya existían 11 aldeas cristianas con 2046 almas. Pero en 1678, dichos choles que ya habían sido convertidos se arrepintieron y destruyeron las aldeas. ${ }^{19}$ En el año de 1685, hubo otro intento de convertir a los choles de nuevo al cristianismo, y fray Agustín Cano logró concentrar otra vez una cantidad de choles en el paraje de San Lucas. ${ }^{20}$ Ya en 1688, los choles en San Lucas se rebelaron otra vez, quemando el poblado y regresaron a la selva, de donde los sacaron por la fuerza para asentarlos en el valle de Urran (Baja Verapaz, en la aldea actual de El Chol). ${ }^{21}$ En 1695 finalmente, se organizó desde Chiapas, Yucatán y Verapaz un ataque combinado contra los pueblos del Petén y del Este de Chiapas que todavía no habían sido sometidos. Fray Agustín Cano acompañó a la última expedición militar bajo el mando de Juan Díaz de Velasco y otra vez lograron convertir una cantidad mayor de choles $^{22}$ antes de seguir adelante hacia la región vecina de Mopanes. Al respecto tenemos en la biblioteca nacional de Guatemala el informe original de Cano que por cierto no es del todo legible en todas sus partes. Las citas que aquí nos interesan son las siguientes: "Pasando la provincia del Chol, que desde Cahabon tiene cuarenta y cinco leguas o cincuenta de atravesia, llegamos a otra nueva nación que se dice de los Mopanes, donde nunca auian mirado Españoles ni ministros del Sto. Euangelio: y aunque la diversidad de la lengua fué de algun embarazo, nos quizo Dios, que hallamos algunos indios Mopanes, que entendían la lengua Chol y por medio de estos logramos el fin de nuestro viaje, el cual que por entonces se logró en algunos adultos, que estando en peligro pidieron el Sto. bautismo y en algunos niños enfermos, que ofrecieron sus padres y fueron al cielo por primicios de aquesta nación. El Cacique principal llamado Taxim Cham huió de nosotros... Mas pacificamos otros 4 casiques de esta nación de Mopanes, llamados en su gentilidad el Cacique Zac, el cacique Yahcab, el cacique Zuzben y el cacique Tezecum".

"Desde Cahobon hasta la Laguna de el Ahiza ay nouenta leguas... en las quarenta y cinco leguas primeras se camina de Cahabon para el Nordeste aunque con varios bueltos: todo esto pertenece a la Provincia de el Chol que se estiende por el oriente hasta las costas de el mar y por la parte de el poniente llega hasta el poderoso rio Xocmó (=Chajmayic) que parece distinto del rio Lacandon, porque este se forma de los vertientes de las Sierras de Zacapulas y entra en la mar por la barra Tabasco y el Xocmó se forma de todos los vertientes que ay desde Cahabon hasta la Laguna de el Ahiza y entra en el mar por la Laguna de Terminos". (Se ve de ahí que Cano ignoraba la confluencia de los dos ríos en el Usumacinta). "Tendra de largo esta Provincia del Chol desde el rio Xocmó hasta el mar cosa de cien leguas”.

"Las otras quarenta y cinco leguas de el Mopan a la laguna se camina de Sur a Norte, con alguna poca declinación al norueste. Esto pertenece a los Mopanes y Ahizaes y se estiende esta tierra por la parte de el Oriente hasta las Costas de el mar y hasta confinar con la peninsula de Yucatan... por parte de el poniente tiene por lindero el mismo rio Xocmo que alli tiene otro nombre" (el presunto río Cancuén, como lo llaman actualmente los indios, mientras que los ladinos lo conocen como río de la Pasión). "Todo el camino de el Mopan á la Laguna es tierra mas tratable; pocos cerros, y no muy altos; las montañas no son tan espesas y se alternan con pinales y campos". Con base en esta descripción se deduce que los mopanes tenían su sede en la región de San Luis y 
podemos suponer entonces que el dialecto de San Luis y San Antonio se derivó del antiguo mopán. Acerca de los mismos mopanes nos dice Cano: "Reconocimos en esta nacion muy poca sinceridad y que tenian intelligencias con los indios de Ahizaes de la Laguna y aun entendimos, que todos ellos eran de una misma nacion itza llamandose Mopan Jtza, Peten Jtza, y que estos Mopanes estauan sujetos al Reyezuelo de la isla de la Laguna". Actualmente el nombre de la parte superior del Río de Belize, Río Mopan, todavía evoca a esta nación.

En el año 1696 fue reubicada de nuevo una cantidad de choles en el paraje Belén en el valle de Urran, mientras que el capellán de Castillo se encargó de trasladar unos 85 choles al paraje Amatique. ${ }^{23}$

Estos informes sobre la colonización de Cobán en conjunto con los relatos arriba mencionados del padre Alonso de Escobar, los cuales están basados en información antigua nos dejan inferir que la reubicación de tribus enteras en otras localidades y espacios diferentes fuese una política común de los conquistadores y misionarios españoles $\mathrm{y}$, de hecho, la tradición oral entre los indios kekchí todavía habla de tales reubicaciones. Por ejemplo en el Norte de Verapaz, me dijeron de manera muy categórica que los habitantes de San Agustín Lanquín habían vivido anteriormente en el sitio de Pec San Agustín. Parece que solamente en tiempos posteriores los españoles empezaron a reubicar a la gente por la fuerza en localidades muy lejanas de sus lugares de origen, como fue el caso de la reubicación efectuada desde la región chol hacia el Valle de Urran en Baja Verapaz. Aparentemente al inicio de la evangelización los españoles consideraron suficiente concentrar a los indios en sitios más cercanos, en donde ellos se convirtieron rápidamente, cuando menos de manera superficial, pero continuaron clandestinamente con sus ritos paganos, viajando a las selvas vírgenes y ofreciendo a sus antiguos dioses paganos los mismos sacrificios de antes. Esto constataron los frailes Francisco Gallegos y Joseph Delgado en su viaje en pro de su misión evangelizadora a la región chol en $1675 .{ }^{24}$ Si rehusamos aceptar la veracidad de las tradiciones orales y las informaciones históricas disponibles, hay apellidos que están atestados con nombres choles y lacandones y que indican la migración de dichas etnias desde el Norte. ${ }^{25}$ Además atestiguan varios de los títulos indígenas de propiedad de tierra en Alta Verapaz expresamente la inmigración de lacandones, por ejemplo un título del año 1539 (?) que se encuentra guardado en San Pedro Carcha y del cual tengo una copia. Los lacandones y acalanes que fueron asentados en Alta Verapaz (especialmente en Cobán), una región que se supuso densamente poblada por indios kekchí, no sólo dejaron de hablar su propia lengua a favor de la de los kekchí dominantes, sino también perdieron poco a poco sus particularidades étnicas. Esto fue un proceso que aún no había terminado en la primera mitad del siglo XIX, según el testimonio de Alonso de Escobar. Me parece, sin embargo, que en las tierras bajas de Lanquín y Cahabon que presuntamente fueron poco pobladas por indios kekchí, los choles reubicados allí dejaron de hablar su lengua, pero no perdieron totalmente sus particularidades étnicas. Al transitar estas regiones a pie, me llamó de inmediato la atención ver que entre los indios kekchí viviera gente que hablara el mismo kekchí, pero que se distinguía de ellos en cuanto a sus características somáticas y etnológicas. ${ }^{26}$

\section{Nombres propios}

José Chamam

Manuel Pou

Domingo Pop

Domingo Pou

Manuel Caal

Kalem Yat

Juan Chamam

Tomas Chamam

\section{Apodos}

Aj uch = cazador del tlacuache

Pok $=$ arena volcánica

Chim $=$ trampa

Cap $=$ casa

Sis $=$ pisote, tejón

$C h a=$ ceniza

Marimb $=$ marimba

$\mathrm{Tu}=$ chichi 
Manuel Pop

Domingo Caal

Juan Pop

Miguel Sel

José Chamam

Domingo Yat

Pedro Yat

Luis Yat

$$
\begin{aligned}
& \text { Camenak = cadáver } \\
& \text { Sac-i-cyuink = hombre } \\
& \text { blanco } \\
& \text { Cuc }=\text { ardilla } \\
& \text { Tap = cangrejo } \\
& \text { K'ot = excrementos } \\
& \text { Ki = dulce } \\
& \text { Chaquicar = pescado seco } \\
& \text { Ma iun bil = ni siquiera llegó al } \\
& \text { lugar del primer descanso (los } \\
& \text { indios kekchí tienen áreas de } \\
& \text { descanso cada determinada } \\
& \text { distancia de camino, llamado } \\
& \text { bil, y con este apodo se refiere } \\
& \text { a la persona que ya descansó } \\
& \text { a su primera salida después } \\
& \text { de la boda antes de llegar } \\
& \text { hasta este lugar. }
\end{aligned}
$$

Mi conclusión de todo esto es que se trata de un grupo étnico que se vio forzado por alguna necesidad social a dejar de hablar su lengua materna. Pienso que dicha necesidad la encontramos en la reducción forzada de los choles a sus lugares de residencia actual.

Voy a recapitular brevemente las diferencias más importantes que hay entre los verdaderos indios kekchí de un lado, y lanquineros y cahaboneros del otro. Lo que no me parece esencial es una pequeña distinción lingüística entre los dialectos hablados; llama más la atención la manera de hablar que es más lenta y melódica entre los cahaboneros y lanquineros, más que sus características somáticas, atuendo y corte de cabello que los distingue. Tampoco entraré en discusión de tales diferencias porque se encuentran a menudo entre cualquier habitante de zonas y aldeas distintas. Más importante son las diferencias que existen entre las autoridades de los verdaderos indios kekchí y de los cahaboneros en Chaal y Cahabon. Además se distinguen notoriamente las construcciones de casas y costumbres funerarias, así como los hábitos culinarios. A la gente de Cahabon, Lanquín y Chisec les gusta comer la carne de ciertas serpientes, por ejemplo otooi y ahauchan, mientras que a los indios kekchí les da asco este tipo de comida.

Desde que inicié mis viajes sospechaba que los cahaboneros y los lanquineros eran reductos de población chol y esta suposición fue clara para mí, cuando mis cargadores kekchí entraron en la primera aldea chol en Tabasco — muy lejos de sus lugares de residencia - y se quedaron con la boca abierta y con exclamaciones de sorpresa. Sin esperar que yo les iba a preguntar al respecto, me comentaron que las mujeres de este lugar se parecían completamente a las de Lanquín. De hecho no solamente se parecen en su tipo somático, sino también en su atuendo; igualmente hay un patrón de tejido del traje masculino que es muy parecido al de ellos, así como la construcción de las casas y los productos de alfarería. El peinado femenino muestra ciertas semejanzas, pero también diferencias, por ejemplo, los gruesos mechones de cabello que les cae sobre sus orejas (llamado laubken kekchî) semejan más bien el peinado de los indios chortí.

Igual que los lanquineros y cahaboneros, la gente de Chisec tiene sus particularidades que indican que no son verdaderos indios kekchí sino un elemento étnico asimilado, aunque igualmente de origen chol. Comparado con esto, no hay nada que recuerde la descendencia de los habitantes de la aldea El Chol en el Valle de Urran excepto su nombre, y por eso tampoco hay indios en este lugar, únicamente mestizos (ladinos).

Como conclusión de las reflexiones aquí compartidas resulta que no podemos tener la claridad absoluta sobre las cuestiones pendientes, pero es probable que los lacandones y los acalanes hablaran chol en los siglos XVI y XVII y por ende el área de difusión de la lengua chol en aquellos tiempos está correctamente indicada en el mapa de Stoll. 
Encontramos entonces los restos de la población chol en el Norte y Este de Guatemala en los lanquineros, los cahaboneros y los chisequeños, mientras que los choles de Honduras ya han desaparecido por completo. Por lo mismo habían desaparecido los lacandones cholanos en el Este de Chiapas que fueron remplazados por mayas inmigrantes del Petén a los cuales se conoce como lacandones, un nombre que se refiere más bien al lugar donde viven y no a su etnicidad. Este nombre no se conoce entre los lacandones quienes se autonombran igual que los mayas: maséwal, y distinguen sus linajes particulares con nombres de animales totémicos. ${ }^{27}$

El número de chisequeños, lanquineros y cahaboneros asciende aproximadamente a 10000 personas, el de los choles y los chortíes a más o menos 20 000, así que el pueblo entero debe contar, en todas sus ramas, con aproximadamente 50000 habitantes. Todos ellos viven una vida retirada y humilde y se alimentan principalmente de los productos que cultivan. Se caracterizan por un alto sentido de libertad y relativamente pocos de ellos trabajan como asalariados en las fincas de los blancos y mestizos. Consecuencia de esto es que en el área chol, los mozos de las fincas son, en su mayoría, indios tzeltales, mientras que los choles prefieren ganar el poco dinero que necesitan para comprarse vestimenta y comida, con su trabajo en su propia tierra. Esto también es resultado del hecho de que en Chiapas no hay ninguna presión sobre los indios para hacer trabajos forzados.

Acerca de los usos y costumbres de los choles y chortíes sabemos, lamentablemente, casi nada, y sobre los hábitos de vida y las particularidades etnológicas de los lanquineros y los cahaboneros tampoco se ha publicado mucho, con excepción de los escasos informes míos que aquí menciono. ¡Esperamos que pronto se animen otros investigadores a llevar a cabo la laboriosa tarea de observar y recabar la cultura original $<$ material e intelectual $>$ que todavía encontramos en estos pueblos! No hay ninguna duda de que a pesar del aislamiento de estos pueblos sus particularidades van a desaparecer paulatinamente, $y$, ¡más tarde podemos esperar solamente un postscriptum de escasos resultados!

Los nombres de las etnias y de lugares mencionados en este texto o en obras históricas disponibles, se encuentran en el esbozo de un mapa; para los casos en los cuales la ubicación del lugar no es precisa, pusimos un signo de interrogación para señalarlo.*

Para ilustrar la gran semejanza que existe entre la lengua chol de Chiapas y Tabasco y la lengua chortí del Este de Guatemala, incluimos un vocabulario, en el cual tomamos en cuenta la lengua chol y chortí, pero también el dialecto pokomam de Jilotepec, porque hasta la fecha dicho dialecto ha sido identificado como chortí según el pequeño vocabulario que nos dejó Stephens. El material lingüístico presentado aquí proviene de mis propias pesquisas que efectué en Tila y en Alianza cerca de Tumbalá para el chol, en Hacienda Grande (Departamento de Cobán, Honduras) para el chortí, así como en El Obraje, Jocotan, Quetzaltepeque (Departamento de Chiquimula, Guatemala) y San Luis Jilotepeque para el pokomam. Desgraciadamente, sólo puedo presentar vocabularios, dado que los indios que encontré en estos lugares en general eran poco capaces de hablar el español y además carecían de la inteligencia para responder correctamente a mis preguntas durante el poco tiempo que me quedaba con ellos. Sin embargo, en las áreas chortí y pokomam logré apuntar algunas conjugaciones, de las cuales en seguida voy a dar unos ejemplos. El vocabulario chol de Juan Josef de la Fuente Albores (Tila, 20 de enero de 1789), depositado en la biblioteca nacional de Guatemala, que lamentablemente no he copiado, y por ello no puedo utilizarlo aquí, lo mismo para el vocabulario choltí del fray Francisco Morán del año 1695. 


\section{Vocabularios chortí, chol y pokomam de Jilotepeque}

Nota: La ortografía utilizada es la española con las modificaciones propuestas por Otto Stoll (Etnografía, p. 39 ss.); " $x$ " suena como "sch" en alemán.*El énfasis siempre descansa sobre la última sílaba. Con las siguientes siglas estoy designando el lugar de acopio: para el chortí - $\mathrm{H}=$ Hacienda Grande; $\mathrm{O}=$ El Obraje; $\mathrm{Q}=$ Quetzaltepeque; $\mathrm{J}=$ Jocotan; para el $\operatorname{chol} \mathrm{T}=$ Tila; $\mathrm{A}=$ Alianza Puyip cerca de Tumbalá.

\begin{tabular}{|c|c|c|c|}
\hline ESPAÑOL & CHORTÍ & CHOL & POKOMAM de JILOTEPEQUE \\
\hline hombre & vuinik $\mathrm{H} \mathrm{Q}$ & vuinik A $\mathrm{T}$ & mas \\
\hline mujer & ixik H Q & ixik A $\mathrm{T}$ & ixok \\
\hline esposo & noxib $\mathrm{O} Q \mathrm{Q}$ & nojxial A & $?$ \\
\hline esposa & ixcar O Q J & yijnam $\mathrm{A}$ ijnab $\mathrm{T}$ & $?$ \\
\hline padre & tat $\mathrm{H} \mathrm{Q}$ & tyat $\mathrm{A}$ tyabá $\mathrm{T}$ & tat \\
\hline madre & tu $\mathrm{H} \mathrm{Q}$ & nya $\mathrm{A}$ babanya $\mathrm{T}$ & tut \\
\hline $\begin{array}{l}\text { hijo (cuando habla } \\
\text { el padre) }\end{array}$ & onen $\mathrm{H} \mathrm{Q} \mathrm{J} \mathrm{O}$ & & \\
\hline $\begin{array}{l}\text { hijo (cuando habla } \\
\text { la madre) }\end{array}$ & ar $\mathrm{H} \mathrm{Q} \mathrm{J} \mathrm{O}$ & halobil A yalobil & ac'un \\
\hline hija & ix chok & $i x$ chok A & pi'usun \\
\hline hermano mayor & sac'un $\mathrm{H}$ itjan $\mathrm{Q} \mathrm{J} \mathrm{O}$ & öscun A & as \\
\hline hermano menor & ijtz'in $\mathrm{H}$ Q J O & ijtz'in A & cha'k \\
\hline hermana mayor & sacun $\mathrm{H} Q \mathrm{Q}$ sacum $\mathrm{O}$ & chich A & as \\
\hline hermana menor & ijtr’in $\mathrm{H} \mathrm{J}$ kumix $\mathrm{O}$ & ijtr'in A & cha'k \\
\hline yerno & nier $\mathrm{H} \mathrm{O} \mathrm{J}$ niar $\mathrm{Q}$ & nijal A & $\ddot{j i}$ \\
\hline nuera & $\operatorname{arib} \mathrm{H} O \mathrm{O} \mathrm{Q}$ & nijal A & ilim \\
\hline cuñado & jan $\mathrm{Q}$ & an $\mathrm{A}$ jaan $\mathrm{T}$ & bakuc \\
\hline cuñada & $n u \mathrm{H}$ jan $\mathrm{Q}$ & $m u \mathrm{~A} \mathrm{~T}$ & ixnap \\
\hline suegro & pixam $\mathrm{H} O \mathrm{O}$ & nijal A & churukijal \\
\hline suegra & pixam $\mathrm{H} \mathrm{O} \mathrm{Q} \mathrm{J}$ & nijal A & tirukija \\
\hline tio & mamá $\mathrm{H}$ Q J O & ichan A yichan $\mathrm{T}$ & $i c a n$ \\
\hline tia & kojná H Q J & $\sin \mathrm{A}$ ichac $\mathrm{T}$ & $(t u r) i$ can \\
\hline abuelo & $\begin{array}{l}\text { tatá viejo } \mathrm{H} \mathrm{O} \mathrm{J} \\
\text { tatá señor } \mathrm{Q}\end{array}$ & inojtiel A nyá $\mathrm{T}$ & mam, mama \\
\hline abuela & tiulamá $\mathrm{H} \mathrm{O} \mathrm{Q}$ & inojitiel $\mathrm{A}$ nyá $\mathrm{T}$ & titu \\
\hline cuerpo & k'erar J Q erar O & puchelá A & nimal \\
\hline hueso & baker $\mathrm{O}$ bak. $\mathrm{J}$ & bak A bökal $\mathrm{T}$ & vuak \\
\hline carne & vuer $\mathrm{H} O \mathrm{O} \mathrm{Q}$ & bökyal A böktyar $\mathrm{T}$ & ch'ak \\
\hline sangre & ch'icher Q O ch'ich J H & ch'ich A ch'ichar $\mathrm{T}$ & qu'ie \\
\hline cabello & tzutzer H O Q J & trutzel A trutre $\mathrm{T}$ & runá, nahis \\
\hline cabeza & jor $\mathrm{Q} \mathrm{O} \mathrm{J}$ or $\mathrm{H}$ & kol A jol $\mathrm{T}$ & jalom \\
\hline frente & $\operatorname{lax} \mathrm{H}$ & pain A bam $\mathrm{T}$ & vuach \\
\hline
\end{tabular}


D O C U M E TOS

\begin{tabular}{|c|c|c|c|}
\hline ESPAÑOL & CHORTÍ & CHOL & POKOMAM de JILOTEPEQUE \\
\hline ojo & $\begin{array}{l}\text { na'ut } \mathrm{O} \text { nak (ni) } \\
\text { ut } \mathrm{H} \mathrm{Q}\end{array}$ & ut $\mathrm{A}$ vut $\mathrm{T}$ & na'kach \\
\hline cejas & majtz'o $\mathrm{H}$ & $\begin{array}{l}\text { mötzab A } \\
\text { muytzab } \mathrm{T}\end{array}$ & 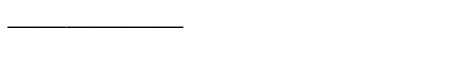 \\
\hline oreja & chiquin $\mathrm{H} \mathrm{O} \mathrm{J} \mathrm{Q}$ & chiquin $\mathrm{A} \mathrm{T}$ & xiquin \\
\hline nariz & $n i \mathrm{H} O \mathrm{Q} \mathrm{J}$ & ni $T$ A & jo \\
\hline boca & $t i \mathrm{H} O \mathrm{O} J$ & $t i \mathrm{~T} \mathrm{~A}$ & chi, evuaré \\
\hline lengua & $a^{\prime} k \mathrm{H} O \mathrm{O} \mathrm{J}$ & k'an $\mathrm{T}$ a'k A & $a^{\prime} k$ \\
\hline incisivo & é $\mathrm{H}$ Q O I & $z e^{\prime} \mathrm{T}$ é $\mathrm{A}$ & $\dot{e}$ \\
\hline muela & ch'am H Q & ch'aam A uché $\mathrm{T}$ & $k a$ \\
\hline barba & tructi $\mathrm{H}$ Q O J & tructi $\mathrm{T} \mathrm{A}$ & $\operatorname{sinchi}$ \\
\hline cuello & nuk & bic $\mathrm{T} A$ & alak \\
\hline nuca & nuk H Q J tzunuk $\mathrm{O}$ & te bic $\mathrm{T} \mathrm{A}$ & alak \\
\hline brazo & $k^{\prime} a p \mathrm{H}$ Q J $a b \mathrm{O}$ & $k^{\prime} \ddot{o b}$ A k'üp T & k'am \\
\hline codo & $\begin{array}{l}\text { usu }(n i) \text { k'ab H Q } \\
\text { amas J }\end{array}$ & $\begin{array}{l}\text { kujk'ub T } \\
\text { xuköb A }\end{array}$ & tr.ic \\
\hline dedo & uni (ni) k'abeh Q & u k'üp T & re'kun (ru) k'am \\
\hline uña & $\begin{array}{l}\text { uni (ni) k'ab O om k'ab J } \\
\text { ejcbak H Q O J }\end{array}$ & $\begin{array}{l}\text { yalol aj k'öb A } \\
\text { ejchak T A }\end{array}$ & ix cák \\
\hline senos & $\operatorname{chu} \mathrm{H} Q \mathrm{O} \mathrm{J}$ & $\operatorname{cbu} \mathrm{A}$ & ch'uch \\
\hline vientre & nak $\mathrm{H} \mathrm{Q} \mathrm{O} \mathrm{J}$ & ni äk. A nük $\mathrm{T}$ & pam \\
\hline ombligo & mok $\mathrm{H}$ Q muk $\mathrm{O} \mathrm{J}$ & & itrum \\
\hline corazón & $\overline{\text { sïmph } \mathrm{O}}$ & pusical A & $\operatorname{sen}(r i) \operatorname{se}$ \\
\hline $\begin{array}{l}\text { Intestinos } \\
\text { pierna }\end{array}$ & $a \mathrm{H} \mathrm{J}$ okob Q O & $\begin{array}{l}\text { soytia } \mathrm{A} \\
\text { ya } \mathrm{A} \mathrm{T}\end{array}$ & (nim ru) tuke. \\
\hline rodilla & pix Q O J & pix $\mathrm{T}$ pökilja A & na chejk \\
\hline pie & ok H Q J okob O & yok $\mathrm{T}$ treniek $\mathrm{A}$ & (perepi ru) tuk \\
\hline sudor & purich $\mathrm{H}$ Q J O & pulich A & vaux \\
\hline orin & apich H Q O J & $\longrightarrow$ & am \\
\hline ala & vuich $\mathrm{H}$ Q O J & vuich $\mathrm{A}$ & $x i c$ \\
\hline pluma & tzutzer $\mathrm{H}$ Q O J & $\longrightarrow$ & $i j$ \\
\hline cola & ne $\mathrm{H} Q \mathrm{O} \mathrm{J}$ & $\longrightarrow$ & jer \\
\hline huevo & k'um H Q O J & $\begin{array}{l}\text { tyumut } \mathrm{A} \\
\text { chentyun } \mathrm{T}\end{array}$ & maloj \\
\hline nido & sijk. $\mathrm{H} Q \mathrm{Q} \mathrm{O} \mathrm{J}$ & k'u A & pächicob \\
\hline aldea, paraje & chinam $\mathrm{H} \mathrm{O} \mathrm{Q} J$ & tum $\mathrm{T}$ tejclum $\mathrm{A}$ & tenamit \\
\hline casa & otot $\mathrm{H}$ O Q J & otot $\mathrm{T}$ otyoy $\mathrm{A}$ & pat \\
\hline pilar & oy $\mathrm{H} \mathrm{O} \mathrm{J}$ & oy A $\mathrm{T}$ & ch'it \\
\hline pared & unayotot $\mathrm{H}$ sarum $\mathrm{J}$ & sacti $\mathrm{T}$ & $k^{\prime} e$ \\
\hline $\begin{array}{l}\text { puerta } \\
\text { techo }\end{array}$ & oporsikap $\mathrm{H}$ & $\begin{array}{l}\text { baje } \\
\text { picti } \mathrm{T} \text { cucul } \mathrm{A}\end{array}$ & \\
\hline viga & quiri $\mathrm{O}$ & $\overline{ }$ & $\longrightarrow$ \\
\hline guano o palma & $x a n \mathrm{O}$ & chaban $\mathrm{T}$ jam $\mathrm{A}$ & $\bar{l}$ \\
\hline cama & ch'akté H Q O J & ch'ak A & ch'at \\
\hline hamaca & $a p \mathrm{O} \mathrm{J}$ & ap $\mathrm{T} \mathrm{A}$ & $\longrightarrow$ \\
\hline camino & bir $\mathrm{H} O \mathrm{Q} \mathrm{J}$ & bij A & vué \\
\hline
\end{tabular}




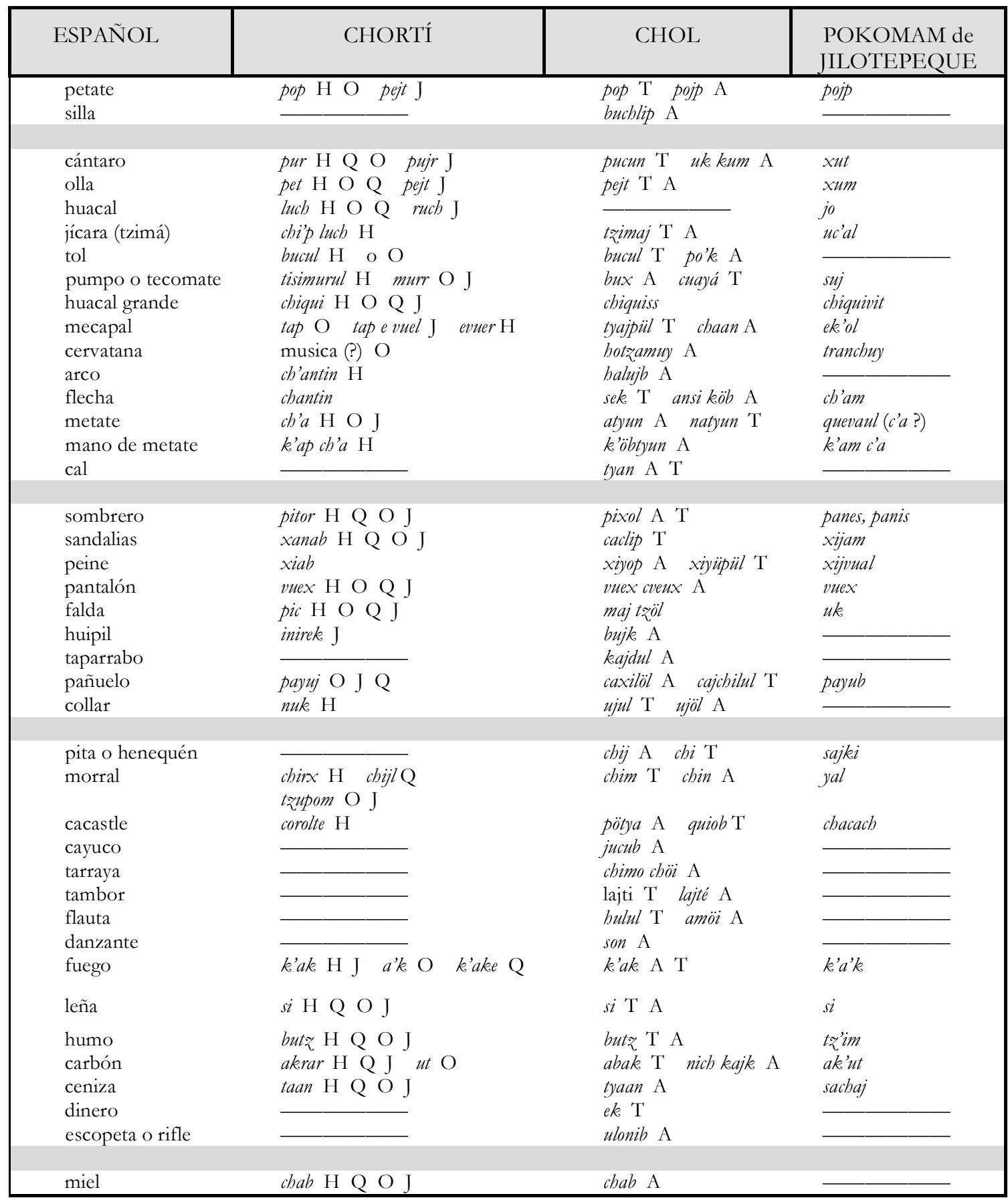


DOCUMENTOS

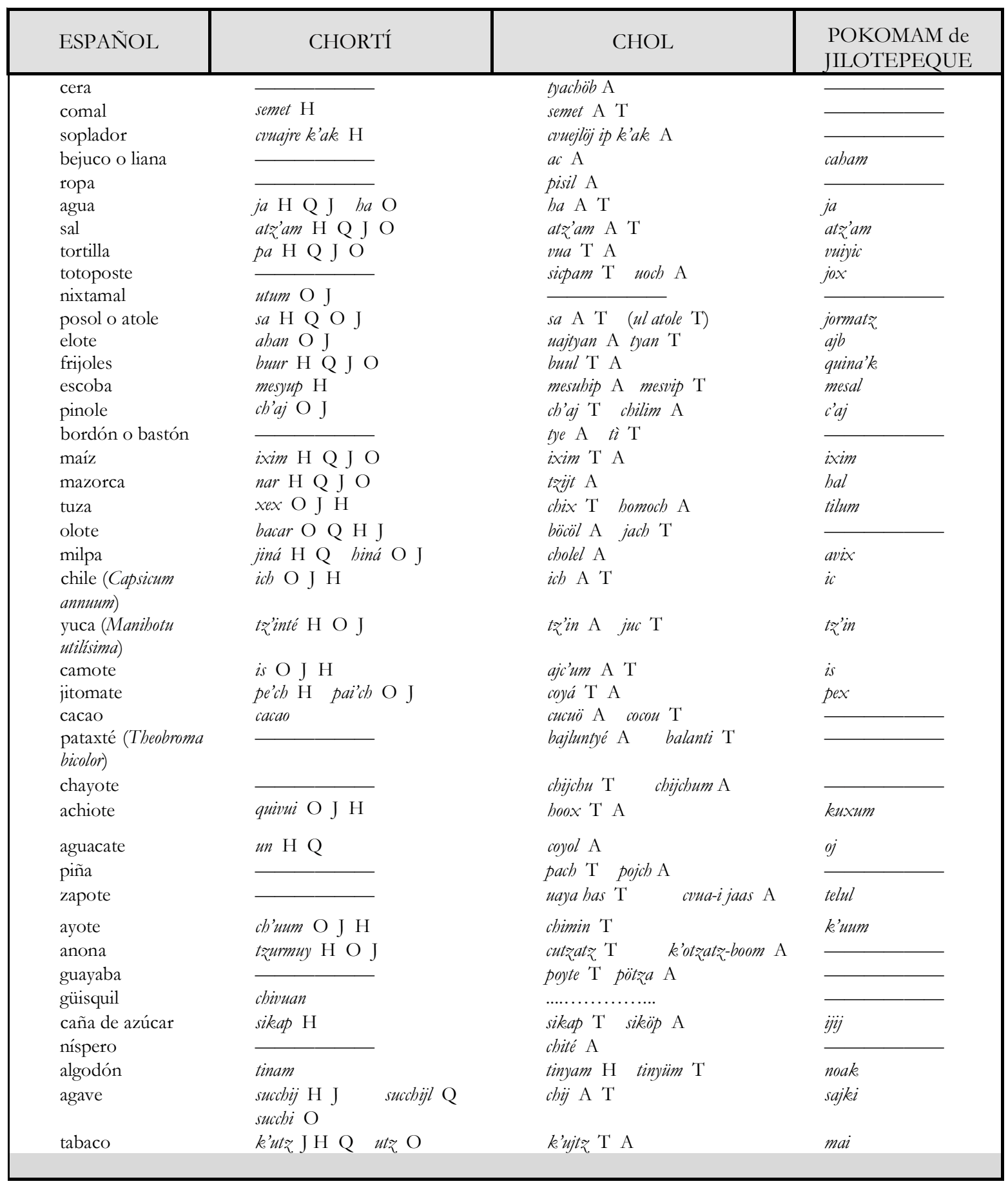


D O C M ENTOS

\begin{tabular}{|c|c|c|c|}
\hline ESPAÑOL & CHORTÍ & CHOL & $\begin{array}{l}\text { POKOMAM de } \\
\text { JILOTEPEQUE }\end{array}$ \\
\hline árbol & té $\mathrm{H} Q \mathrm{O} \mathrm{J}$ & kyé(?) $\mathrm{T}$ tyé $\mathrm{A}$ & chè \\
\hline hoja & yopor H Q O J & yopom $\mathrm{T}$ A & xak \\
\hline rama & (u) k'ab e tè H Q J & k'anax tyé $\mathrm{T}$ & k'am \\
\hline flor & nichir $\mathrm{H}$ & nichtyé A & piché \\
\hline espina u ortiga & quiix $\mathrm{H}$ Q O J & ch'ix T A & qu'ix \\
\hline raíz & vuir $\mathrm{H} \mathrm{O} \mathrm{J}$ & chac $\mathrm{T}$ vitjé $\mathrm{A}$ & (risbal nyo) \\
\hline plátano & jass $\mathrm{H} \mathrm{O} \mathrm{J} \mathrm{Q}$ & haas $\mathrm{T}$ jaas $\mathrm{A}$ & pulak \\
\hline pino & taté $\mathrm{H}$ ta $\mathrm{O}$ & tyaj $\mathrm{T} \mathrm{A}$ & chaj \\
\hline & taj te J taj Q & haam A hams T & \\
\hline $\begin{array}{l}\text { zacate } \\
\text { caoba }\end{array}$ & $a c \mathrm{O} \mathrm{J}$ & $\begin{array}{l}\text { haam A bamp } \mathrm{T} \\
\text { sutzujl A }\end{array}$ & - \\
\hline cedro & - & chuj tyé A & ـ \\
\hline liquidámbar & $\longrightarrow$ & sutrté A & - \\
\hline helecho & $\longrightarrow$ & strijb A & - \\
\hline acuahal o matorral & k'opot $\mathrm{J}$ opot $\mathrm{O}$ & tiel A matial $\mathrm{T}$ & \\
\hline bosque & 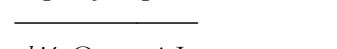 & nojtiel A matial $\mathrm{T}$ & chè \\
\hline sabana & chiá $\mathrm{O} \quad x a j \mathrm{~J}$ & jamil A yatipitz A & tracial \\
\hline chicozapote & $\overline{ }$ & ch'ich A T & \\
\hline índigo & - & chajuc $\mathrm{T}$ & - \\
\hline $\begin{array}{l}\text { palo de Brasil } \\
\text { copal }\end{array}$ & $\bar{\square}$ & $\begin{array}{l}\text { chicté } \mathrm{T} \quad \text { tintyajtyé } \mathrm{A} \\
\text { chajan } \mathrm{T}\end{array}$ & \\
\hline pimiento & $\longrightarrow$ & ichtiol A & \\
\hline puerco & chitam $\mathrm{H}$ Q J O & chityam A $\mathrm{T}$ & $a k$ \\
\hline perro & tzi $i \mathrm{H} \mathrm{J} O$ & tri A T & $t^{\prime} i$ \\
\hline gato & mis $\mathrm{H}$ Q J O & mis A $\mathrm{T}$ & mis \\
\hline conejo & tur $\mathrm{J}$ & ujchip A & emul \\
\hline gallo & $\longrightarrow$ & chityomut A & $a c^{\prime} a c h$ \\
\hline gallina & ac'ach $\mathrm{H} \mathrm{J}$ tuach $\mathrm{O}$ & a mut $\mathrm{A}$ mut $\mathrm{T}$ & tut ac'ach \\
\hline pájaro & mut $\mathrm{O} \mathrm{J} \mathrm{Q} \mathrm{H}$ & chijchip A acxi $\mathrm{T}$ & chicob \\
\hline pavo & $\longrightarrow$ & ajtzo A & $\longrightarrow$ \\
\hline zopilote & usij $\mathrm{H}$ Q J O & usijl A tyabol $\mathrm{T}$ & c'uch \\
\hline venado & masa H Q J O (atzekisch) & $m e \mathrm{~A} \mathrm{~T}$ & quej \\
\hline $\begin{array}{l}\text { cenzo (puerco del } \\
\text { monte) }\end{array}$ & k'octar chitam $\mathrm{J}$ ebitam $\mathrm{H}$ & mate chityam A matichtyam $\mathrm{T}$ & panchamal ak \\
\hline jabalí & - & boyom A balau $\mathrm{T}$ & 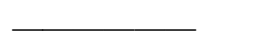 \\
\hline tapir & $\longrightarrow$ & trinú $\mathrm{A} \mathrm{T}$ & 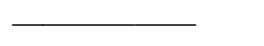 \\
\hline taltusa & pa $\mathrm{H}$ & ba $\mathrm{A} \mathrm{T}$ & cvuá \\
\hline cotusa & $\begin{array}{l}\text { chujbub } \mathrm{J} \text { chujba } \mathrm{O} \text { chupub } \\
\mathrm{H} \text { chuk'ub Q }\end{array}$ & & \\
\hline jaguar & bajram J & bajlum $\mathrm{T}$ A & tiyan \\
\hline pantera, puma & bajaam $\mathrm{O}$ & chöc balum A & $\operatorname{cojl}$ \\
\hline $\begin{array}{l}\text { armadillo } \\
\text { pisote o tejón }\end{array}$ & $\overline{\text { patriuty } \mathrm{H} \mathrm{O} \mathrm{I}}$ & ip & itr'ul \\
\hline ardilla & chuch $\mathrm{H}$ O J & chuch A $\mathrm{T}$ & cuc \\
\hline
\end{tabular}




\begin{tabular}{|c|c|c|c|}
\hline ESPAÑOL & CHORTÍ & CHOL & $\begin{array}{l}\text { POKOMAM de } \\
\text { JILOTEPEQUE }\end{array}$ \\
\hline \multirow{8}{*}{$\begin{array}{l}\text { coyote } \\
\text { chango } \\
\text { mono araña } \\
\text { ratón } \\
\text { murciélago } \\
\text { lagarto } \\
\text { serpiente o culebra } \\
\text { tortuga }\end{array}$} & bojb $\mathrm{H} \mathrm{O} \mathrm{J}$ & \multirow{3}{*}{$\begin{array}{l}\text { k'am bolay } \mathrm{T} \\
\max \mathrm{A} \mathrm{T} \\
\text { batz } \mathrm{T}\end{array}$} & \multirow{3}{*}{$\begin{array}{l}x \text { xojb } \\
\text { c'oy }\end{array}$} \\
\hline & & & \\
\hline & $\longrightarrow$ & & \\
\hline & ch'ok H O Q J & \multirow{5}{*}{$\begin{array}{l}\text { tzuc } \mathrm{A} \\
\text { su'tz } \mathrm{A} \\
\text { ajin } \mathrm{A} \text { abin } \mathrm{T} \\
\text { bucum } \mathrm{T} \text { lucum } \mathrm{A} \\
\text { aje } \mathrm{A} \mathrm{T}\end{array}$} & \multirow{5}{*}{$\begin{array}{l}\text { ch'o } \\
\text { so'tz. } \\
\text { Nijin } \\
\text { May }\end{array}$} \\
\hline & su'tr. $\mathrm{H} \mathrm{O} \mathrm{J}$ & & \\
\hline & $\operatorname{ain} \mathrm{J}$ & & \\
\hline & chan $\mathrm{O} \mathrm{J} \mathrm{H}$ & & \\
\hline & & & \\
\hline pez & chai $\mathrm{H}$ Q J O & chö A chuy $\mathrm{T}$ & \\
\hline cangrejo & hорор O J & mep $\mathrm{T}$ A & ch'om \\
\hline camarón & triktrik O J & xex $\mathrm{T}$ xun $\mathrm{A}$ & tap \\
\hline alacrán & $\operatorname{sinam} \mathrm{H} Q \mathrm{O} J$ & trinay $\mathrm{T} \operatorname{sinian} \mathrm{A}$ & $\sin a j$ \\
\hline mariposa & pejpem H Q J & pejtem T A & sam \\
\hline hormiga & xinich $\mathrm{H}$ Q J O & xinich $\mathrm{T} \mathrm{A}$ & sinic \\
\hline caracol & choch O J & puy T A & pur \\
\hline almeja & peremech $\mathrm{H}$ & bejmech $\mathrm{T}$ bujch $\mathrm{A}$ & loch \\
\hline cucaracha & & pebua $\mathrm{T}$ maco $\mathrm{A}$ & taltapach \\
\hline zancudo & us $\mathrm{H} \mathrm{Q} \mathrm{O} \mathrm{J}$ & $u s \mathrm{TA}$ & utz. \\
\hline mosquito & uchá $\mathrm{H} Q \mathrm{O} \mathrm{J}$ & uch ba T A & uchá \\
\hline araña & am $\mathrm{H} \mathrm{Q} \mathrm{O} \mathrm{J}$ & am $\mathrm{A}$ & $a m$ \\
\hline garrapata & $\operatorname{sip} \mathrm{Q} \mathrm{O} \mathrm{J}$ & $\operatorname{sip} \mathrm{T} \mathrm{A}$ & sïp \\
\hline pulga & $c b^{\prime} a c \mathrm{H} \mathrm{Q} \mathrm{OJ}$ & ch'üc $\mathrm{T}$ ch’öc A & ca'k \\
\hline chinche & ochem ch'ac $\mathrm{H}$ & 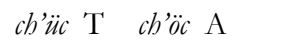 & c'ak \\
\hline piojo & uch $\mathrm{O}$ & uch $\mathrm{T} \mathrm{A}$ & $u c$ \\
\hline gusano & upip H Q upi $\mathrm{O} \mathrm{J}$ & motzo A & sabú \\
\hline \multirow{3}{*}{$\begin{array}{l}\text { quetzal } \\
\text { hocofaisán } \\
\text { avispa }\end{array}$} & $\longrightarrow$ & \multirow{3}{*}{$\begin{array}{l}x \text { mank'uk A } \\
\text { chac mut A } \\
\text { xux A }\end{array}$} & 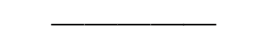 \\
\hline & chac mut A & & $\longrightarrow$ \\
\hline & yair $\mathrm{O}$ yarkir $\mathrm{J}$ & & colax \\
\hline \multirow{12}{*}{$\begin{array}{l}\text { tierra } \\
\text { piedra } \\
\text { arena } \\
\text { mugre } \\
\text { montaña } \\
\text { llanura o meseta } \\
\text { mar } \\
\text { río } \\
\text { gruta o cueva } \\
\text { camino } \\
\text { puente } \\
\text { plata } \\
\text { hierro }\end{array}$} & luum $\mathrm{H} \mathrm{O}$ ruum $\mathrm{Q}$ & \multirow{10}{*}{$\begin{array}{l}\text { luum } \mathrm{A} \mathrm{T} \\
\text { tun } \mathrm{T} \text { tyun } \mathrm{A} \\
\text { bi } \mathrm{T} \text { ji } \mathrm{A} \\
\text { ok'om } \mathrm{T} \text { ok'ol } \mathrm{A} \\
\text { uitz } \mathrm{T} \mathrm{A} \\
\text { boktil } \mathrm{T} \text { oktyil } \mathrm{A} \\
\text { aban } \mathrm{T} \text { ajp } \mathrm{A} \\
\text { ha } \mathrm{T} \text { nojha } \mathrm{A} \\
\text { chintyum } \mathrm{T} \text { ch'en } \mathrm{A} \\
\text { bi } \mathrm{T} \text { bij } \mathrm{A} \\
\text { cajtu } \mathrm{T}\end{array}$} & ac'al \\
\hline & tun $\mathrm{H} Q \mathrm{O} \mathrm{J}$ & & avuaj \\
\hline & bi $\mathrm{H} Q \mathrm{O} \mathrm{J}$ & & sanim \\
\hline & anam $\mathrm{H} \mathrm{Q} \mathrm{O} \mathrm{J}$ & & chuvuá \\
\hline & vuitzir $\mathrm{H} \mathrm{Q} \mathrm{O}$ chen $\mathrm{J}$ & & yuk. \\
\hline & $\longrightarrow$ & & quixcam \\
\hline & $\overline{2}$ & & $\longrightarrow$ \\
\hline & noja H O Q k’o J & & ja \\
\hline & $\overline{\text { bir } \mathrm{H} \mathrm{O} \mathrm{Q} \mathrm{J}}$ & & vué \\
\hline & $\longrightarrow$ & & 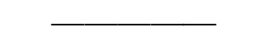 \\
\hline & tumin $\mathrm{H}$ O Q J & tyakin A & puak \\
\hline & takin $\mathrm{H} \mathrm{Q}$ tain $\mathrm{O} \mathrm{J}$ & tzucu tyakin $\mathrm{A}$ & chi'ch \\
\hline
\end{tabular}


D O C M ENTOS

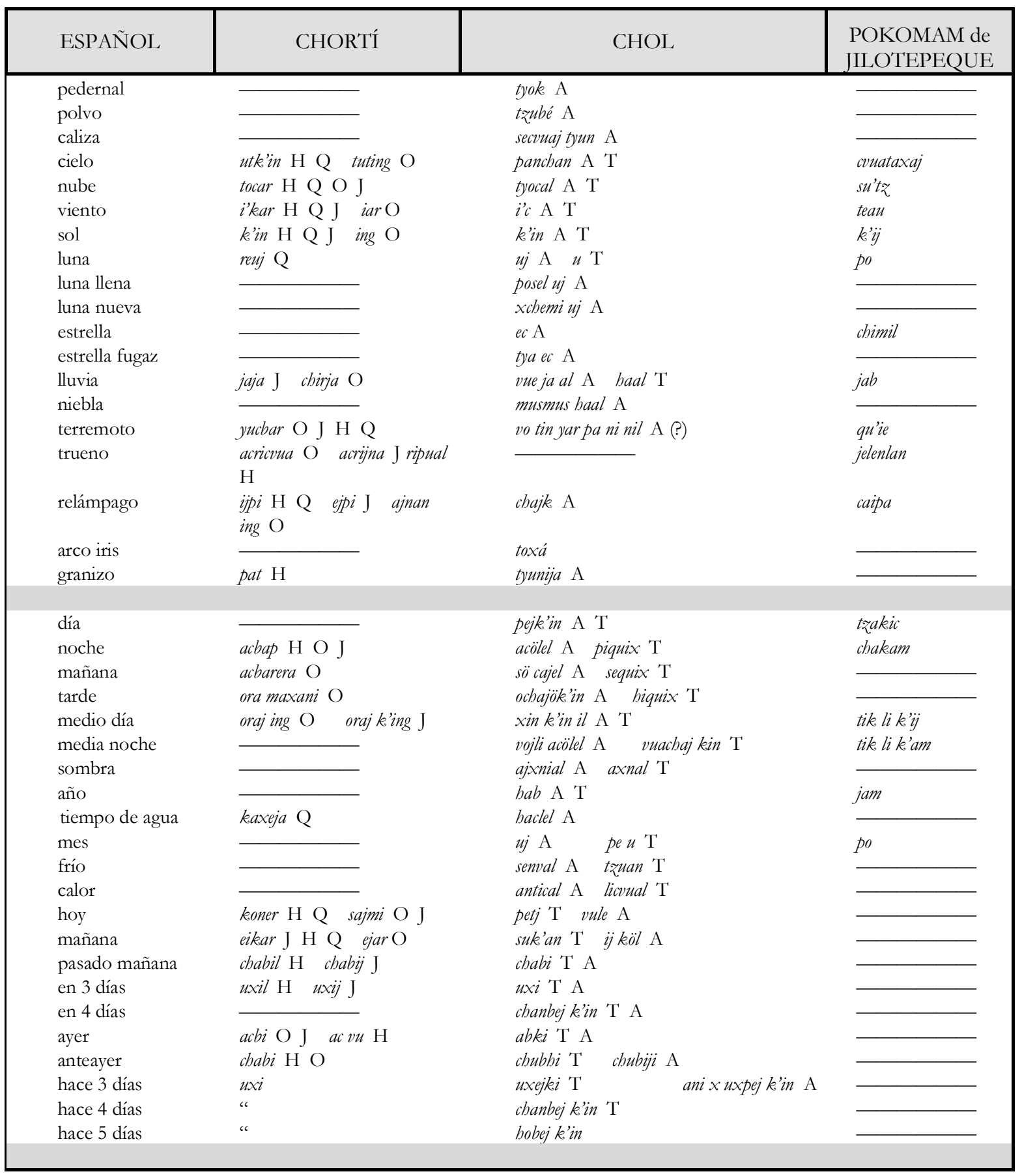


O C UMENTOS

\begin{tabular}{|c|c|c|c|c|}
\hline ESPAÑOL & CHORTÍ & \multicolumn{2}{|c|}{ CHOL } & $\begin{array}{l}\text { POKOMAM de } \\
\text { JILOTEPEQUE }\end{array}$ \\
\hline 1 & inte $\mathrm{J} \mathrm{O}$ & jun (tiqui) $\mathrm{T}$ & jun (bel) A & jenaj \\
\hline 2 & chate $\mathrm{J} \mathrm{O}$ & cha (tquid) $\mathrm{T}$ & $c h a b$ (bed) A & queem \\
\hline 3 & uxte $\mathrm{J}$ uxti $\mathrm{O}$ & ox (tquil) $\mathrm{T}$ & $u x(b e d)$ & ixim \\
\hline 4 & chante $\mathrm{J} \mathrm{O}$ & chin (tquil) $\mathrm{T}$ & chön (bed) A & quejem \\
\hline 5 & & bo (tquil) $\mathrm{T}$ & boob (bed) A & hoom \\
\hline 6 & - & vии (tquil) $\mathrm{T}$ & vnök (bel) A & viakin \\
\hline 7 & - & vuntb (tquil) $\mathrm{T}$ & vunk (bel) A & vunkum \\
\hline 8 & $\longrightarrow$ & vuaxat (tquil) $\mathrm{T}$ & vuajxok (bel) A & vuakxakim \\
\hline 9 & & bolon (tquil) $\mathrm{T}$ & bolon (bel) A & \\
\hline 10 & & doen (tquil) $\mathrm{T}$ & lujum (bel) A & \\
\hline 11 & & najchen (tquil) $\mathrm{T}$ & bujluch (bed) A & \\
\hline 12 & & lajchom (bed) A & & \\
\hline 13 & & uxlijum (bel) A & & \\
\hline 14 & & chonlujum (bel) A & & \\
\hline 15 & & boolujum (bel) A & & \\
\hline 16 & & vuoklujum (bed) & & \\
\hline 17 & & vunklujum (bel) & & \\
\hline 18 & & vuajxoklujum (be & & \\
\hline 19 & & bolonlujum (bel) & & \\
\hline 20 & & jun cal A & & \\
\hline 21 & & junbel i nume ti ju & al A & \\
\hline 22 & & chabel i nume ti ju & cal A & \\
\hline 30 & & lujumbel i cha cal & & \\
\hline 40 & & chacal A & & \\
\hline 50 & & lujumbel i ux cal & & \\
\hline 60 & & uxcal A & & \\
\hline 70 & & lujumtrij ti chonca & & \\
\hline 80 & & choncal A & & \\
\hline 90 & & lijum trij ti boo co & & \\
\hline 100 & & hoocal A & & \\
\hline 120 & & vuök cal & & \\
\hline 140 & & vunk cal & & \\
\hline 160 & & vnajxöke cal & & \\
\hline 180 & & boloncal & & \\
\hline 200 & & lijum cal & & \\
\hline 220 & & bujluch & & \\
\hline 240 & & lajchön cal & & \\
\hline 260 & & uxlujum cal, etc. & & \\
\hline 300 & & boo lujum cal & & \\
\hline 400 & & jun bac & & \\
\hline 500 & & boo cal i cha bac & & \\
\hline 800 & & cha bac & & \\
\hline 1000 & & lujum cal i ux bac & & \\
\hline
\end{tabular}




\begin{tabular}{|c|c|c|c|}
\hline ESPAÑOL & CHORTÍ & CHOL & $\begin{array}{l}\text { POKOMAM de } \\
\text { JILOTEPEQUE }\end{array}$ \\
\hline $\begin{array}{l}1200 \\
2600 \\
3000 \\
4000 \\
8000 \\
16000\end{array}$ & & $\begin{array}{l}\text { ux bac } \\
\text { chönbac } \\
\text { hoobac } \\
\text { lujumbac } \\
\text { jun cal ti bac } \\
\text { cha cal ti bac }\end{array}$ & \\
\hline $\begin{array}{l}\text { yo } \\
\text { tú } \\
\text { él } \\
\text { nosotros } \\
\text { vosotros } \\
\text { ellos } \\
\text { mío } \\
\text { tuyo } \\
\text { suyo } \\
\text { nuestro } \\
\text { vuestro } \\
\text { sus } \\
\text { sí } \\
\text { no }\end{array}$ & $\begin{array}{l}\text { nen } \mathrm{O} \\
\text { net } \mathrm{O} \\
\text { baxir } \mathrm{O} \\
\text { non } \mathrm{O} \\
\text { nox } \mathrm{O} \\
\text { haxirub } \\
\text { ni (prefijo antes de consonantes) } \\
a- \\
u- \\
k a- \\
i- \\
u \ldots(\mathrm{ob}) \\
\text { bubu } \mathrm{O} \\
\text { maachi } \mathrm{O}\end{array}$ & $\begin{array}{l}\text { la } \\
\\
\text { nivu (prefijo antes de vocales) } \\
v u \ldots \\
\text { иy... } \\
\text { kavu... } \\
\text { y... } \\
\text { uy... (ob) }\end{array}$ & $\begin{array}{l}\text { ben } \\
\text { bet } \\
\text { re } \\
\text { koj } \\
\text { beta } \\
\text { retaquè }\end{array}$ \\
\hline
\end{tabular}


Ejemplo de conjugaciones en chortí, El Obraje

\begin{tabular}{|c|c|c|c|c|c|c|c|}
\hline \multicolumn{2}{|c|}{ PRESENTE } & \multicolumn{2}{|c|}{ PRETÉRITO } & \multicolumn{2}{|c|}{ FUTURO } & \multicolumn{2}{|c|}{ IMPERATIVO } \\
\hline voy & in xin & fui & vixien & iré & ompa & & \\
\hline vas & $i x i n$ & fuiste & vixiet & irás & ixiopa & & \\
\hline va & $a x i n$ & fue & vixin & irá & axaupa & & \\
\hline vamos & kaxin & fuimos & vixion & iremos & kaupa & & \\
\hline $\begin{array}{l}\text { ustedes } \\
\text { van }\end{array}$ & $i x \ddot{i x i n}$ & fueron & vixiox & irán & ixiopa & & \\
\hline ellos van & $a x i o b$ & fueron & vixiob & irán & axaupob & & \\
\hline vengo & yopenix & vine & yopen & & & & \\
\hline vienes & yopetix & viniste & yopet & & & & \\
\hline viene & yopix & vino & yapa & & & & \\
\hline venimos & poponix & venimos & yopon & & & & \\
\hline vienen & yopoxix & vinieron & yopox & & & & \\
\hline vienen & yopovix & vinieron & yopob & & & & \\
\hline duermo & incvuapan & dormí & cvuayananix & dormiré & auincvuayan & & \\
\hline duermes & icvuayan & dormiste & acvuayanetix & dormirás & ixicunayan & ¡duérmete! & icvuayan \\
\hline duerme & avuayan & durmió & cvuayan & dormirá & achecvuayan & & \\
\hline dormimos & kacunayan & durmimos & couayanonix & dormiremos & anicavnayan & & \\
\hline duermen & ieiicvuayan & durmieron & Cruayanonix & dormirán & ixixincvuayan & & \\
\hline duermen & acvuayan & durmieron & couyanob & dormirán & axicunayanob & & \\
\hline lavo & impojtyi & & & & & & \\
\hline lavas & apojtyi & & & & & & \\
\hline lava & upojtyi & & & & & & \\
\hline lavamos & kapojtyi & & & & & & \\
\hline lavan & ipojtyi & & & & & & \\
\hline lavan & pojtyiob & & & & & & \\
\hline & & calenté & inbajnix & calentaré & auin ijnes & & \\
\hline & & calentaste & abajnix & calentarás & ïnes (?) & ¡calienta! & ïnes \\
\hline & & calentó & ubajnix & calentará & aunijnes & & \\
\hline & & calentamos & kabajnix & calentaremos & kaijnes & & \\
\hline & & calentaron & ibajnix & calentarán & oaviijnes (?) & ¡calienten! & ijnesic \\
\hline & & calentaron & ubajnonix & calentarán & aunijinesob & & \\
\hline
\end{tabular}




\begin{tabular}{|c|c|}
\hline \multicolumn{2}{|c|}{ Ejemplos de unas frases chortí } \\
\hline \multicolumn{2}{|c|}{ Hacienda Grande (Honduras) } \\
\hline Entro en mi casa & inic ta ni otto 28 \\
\hline Voy a Copán & inxic Copan \\
\hline Viné de Pexhá & yopen Pexha \\
\hline Apago el fuego & tajpé te k'ak \\
\hline Entro en la casa de mi padre & oinchoy ti otot ni tata \\
\hline ¿Tu casa es grande? & na ta yotot \\
\hline Mi mujer ha molido el maíz & ni cvuixcar ujjutyu pek.um \\
\hline ¿Hay caracoles en el río? & tyal ochoch ta noja \\
\hline ¿Tienes muchos hijos? & tyal mani cvuarob \\
\hline El agua está fría & insis è ha \\
\hline Los frijoles están sabrosos & intrap è bur \\
\hline Comí los frijoles & incuxik è bur \\
\hline La caña está dulce & inchi è sikab \\
\hline El hombre compró sal & e yuinik umané atzam \\
\hline \multicolumn{2}{|c|}{ El Obraje (Guatemala) } \\
\hline La gente va al pueblo para comprar sal & vixin e vuinik ta chinam umani atram \\
\hline ¿Dónde está el hombre? & tyaxan e yuinik. \\
\hline ¡Ven acá! & lar tara \\
\hline En mi casa tengo mi maíz & ta nicruotot hayan ni nar \\
\hline $\begin{array}{l}\text { Mi mujer ha preparado la masa para hacer las } \\
\text { tortillas en el comal, pero el perro se las comió }\end{array}$ & $\begin{array}{l}\text { ni cvuixcar ujuchix u keum u muxmux e pa ta semet } \\
\text { u uxi e pa e tri }\end{array}$ \\
\hline
\end{tabular}


Ejemplo de conjugaciones en pokomam de Jilotepeque

\begin{tabular}{|c|c|c|c|c|c|c|c|}
\hline \multicolumn{2}{|c|}{ PRESENTE } & \multicolumn{2}{|c|}{ PRETÉRITO } & \multicolumn{2}{|c|}{ FUTURO } & \multicolumn{2}{|c|}{ IMPERATIVO } \\
\hline caliento & nupajrij & calenté & inupajrij & calentaré & $\begin{array}{l}\text { nanvaja } \\
\text { nupajonrij }\end{array}$ & & \\
\hline calientas & naparij & calentaste & hapajonrij & calentarás & $\begin{array}{l}\text { nanvaja } \\
\text { happajonrij }\end{array}$ & & \\
\hline calienta & empavuirij & calentó & rupajonrij & calentará & $\begin{array}{l}\text { nanroja } \\
\text { rupajonrij }\end{array}$ & & \\
\hline calentamos & emkapajij & calentamos & kapajonrij & calentaremos & nakoje kapajonrij & & \\
\hline calientan & napaj tarij & calentaron & bapajonrij & calentarán & $\begin{array}{l}\text { natavoja } \\
\text { hapajonrij }\end{array}$ & & \\
\hline calientan & emquepajrij & calentaron & quipajonrij & calentarán & nacojè quipajonrij & & \\
\hline lovo & & & & Llouré tó & D & & \\
\hline lavo & intrajune & lave & nutzajom & lavare & navaje nutzajom & & \\
\hline lavas & natrajom & lavaste & hatzajom & lavarás & navaje hatagjom & & \\
\hline lava & utraj & lavó & rutzajom & lavará & naroju rutazajom & & \\
\hline lavamos & inkatraj & lavamos & hatzajom & lavaremos & nakojé hatzajom & & \\
\hline lavan & natraj & lavaron & hatrajom & lavarán & $\begin{array}{l}\text { natanvaje } \\
\text { hatrajom }\end{array}$ & & \\
\hline lavan & quitzajvu & lavaron & quizajom & lavarán & nacoje quitrajom & & \\
\hline quiero escribir & $\begin{array}{l}\text { bincvua } \\
\text { kinsirvé }\end{array}$ & quise escribir & $\begin{array}{l}\text { ejuna } \\
\text { rexinsirvé }\end{array}$ & querré escribir & nanvoje sirvo & & \\
\hline $\begin{array}{l}\text { quieres } \\
\text { escribir }\end{array}$ & $\begin{array}{l}\text { navua } \\
\text { hensirvé }\end{array}$ & $\begin{array}{l}\text { quisiste } \\
\text { escribir }\end{array}$ & $\begin{array}{l}\text { xanvua } \\
\text { etisirvé }\end{array}$ & querrás escribir & nanvoje sirvo & & \\
\hline quiere escribir & binrä hensirvé & quiso escribir & ijra ejsirvé & querrá escribir & naroje sirvo & & \\
\hline $\begin{array}{l}\text { queremos } \\
\text { escribir }\end{array}$ & inkea kasirvé & $\begin{array}{l}\text { quisimos } \\
\text { escribir }\end{array}$ & ejka xajsirvé & $\begin{array}{l}\text { querremos } \\
\text { escribir }\end{array}$ & $\begin{array}{l}\text { nakojeka } \\
\text { rekasirvé (?) }\end{array}$ & & \\
\hline $\begin{array}{l}\text { quieren } \\
\text { escribir }\end{array}$ & $\begin{array}{l}\text { nanvua } \\
\text { tatesirvé }\end{array}$ & $\begin{array}{l}\text { quisieron } \\
\text { escribir }\end{array}$ & $\begin{array}{l}\text { Xanvata } \\
\text { etasirvé }\end{array}$ & querrán escribir & natavoja sirvo & & \\
\hline $\begin{array}{l}\text { quieren } \\
\text { escribir }\end{array}$ & inka quesirvé & $\begin{array}{l}\text { quisieron } \\
\text { escribir }\end{array}$ & ejca xesirvé & querrán escribir & nacojé sirvo & & \\
\hline trenzo & namianom & trencé & ejnoan & trenzaré & novaje noanam & & \\
\hline trenzas & naanom & trenzaste & xaan & trenzarás & navage haanom & ¡trenza! & chanher \\
\hline trenza & narcanom & trenzó & èancvue & trenzará & narage roanom & & \\
\hline trenzamos & nakaanom & trenzamos & ejkaan & trenzaremos & nakojo kaanom & & \\
\hline trenzan & nataanom & trenzaron & xaanta & trenzarán & natavojé anom & ¡trencen! & chantalien \\
\hline
\end{tabular}




\begin{tabular}{|c|c|c|c|c|c|c|c|}
\hline \multicolumn{2}{|c|}{ PRESENTE } & \multicolumn{2}{|c|}{ PRETÉRITO } & \multicolumn{2}{|c|}{ FUTURO } & \multicolumn{2}{|c|}{ IMPERATIVO } \\
\hline trenzan & naqueanom & trenzaron & xeancvue & trenzarán & nacoje & & \\
\hline & & & & & queanom & & \\
\hline soy o estoy & betvuilcat & fui o estuve & Xincvu $^{29}$ & seré o estaré & nanvoje vui & & \\
\hline eres o estás & vuilquin & fuiste o estabas & ticvui & serás o estarás & nanvoje vui & ¡sé! & vuilcat \\
\hline es o está & ville & fue o estaba & coni & será o estará & naroje vui & & \\
\hline $\begin{array}{l}\text { somos o } \\
\text { estamos }\end{array}$ & kojvuilkoj & $\begin{array}{l}\text { fuimos o } \\
\text { estábamos }\end{array}$ & xajvii & $\begin{array}{l}\text { seremos o } \\
\text { estaremos }\end{array}$ & nakove vii & & \\
\hline son o están & tavuilcat & $\begin{array}{l}\text { fueron o } \\
\text { estaban }\end{array}$ & tivuita & serán o estarán & notanvoge vii & ¡sed! & tanvuilcat \\
\hline son o están & vuique & $\begin{array}{l}\text { fueron o } \\
\text { estaban }\end{array}$ & xuvui & serán o estarán & nacoje cvui & & \\
\hline mato & Nacamsa ${ }^{30}$ & maté & enucamsa & mataré & $\begin{array}{l}\text { nanvoja } \\
\text { núcamsam }\end{array}$ & & \\
\hline matas & nacamsa & mataste & xacamsa & matarás & $\begin{array}{l}\text { navajo } \\
\text { hacamsam }\end{array}$ & ¡mata! & cha camsa \\
\hline mata & narucamsa & mató & ejcamsinic & matará & $\begin{array}{l}\text { narojo } \\
\text { camsinoc }\end{array}$ & & \\
\hline matamos & ejkacamsa & matamos & kacamsam & mataremos & $\begin{array}{l}\text { nakojé } \\
\text { kecamsam }\end{array}$ & & \\
\hline matan & nateamsata & mataron & tacamsam & matarán & $\begin{array}{l}\text { natanvojé } \\
\text { hacamsam }\end{array}$ & ¡maten! & $\begin{array}{l}\text { cha camsa ta } \\
\text { het } \\
\end{array}$ \\
\hline matan & binticamsa & mataron & quicamsam & matarán & $\begin{array}{l}\text { nacojé } \\
\text { quicamsam }\end{array}$ & & \\
\hline escucho & bincvuarej & escuché & vuaren & escucharé & $\begin{array}{l}\text { nanvojé } \\
\text { cvuaren }\end{array}$ & & \\
\hline escuchas & nacvuarej & escuchaste & bacvuaren & escucharás & $\begin{array}{l}\text { nanvojé } \\
\text { hanvuaren }\end{array}$ & ¡escucha! & chavuarej \\
\hline escucha & henucvuarej & escuchó & ruvuaren & escuchará & $\begin{array}{l}\text { narojé } \\
\text { ruvuaren }\end{array}$ & & \\
\hline escuchamos & henkacvuarej & escuchamos & kacvuaren & escucharemos & $\begin{array}{l}\text { nakojé } \\
\text { kacvuaren }\end{array}$ & & \\
\hline escuchan & nancvuarej & escucharon & couarenta & escucharán & $\begin{array}{l}\text { natacouojé } \\
\text { navuaren }\end{array}$ & ¡escuchan! & chavuarejta \\
\hline escuchan & quiconirené & escucharon & quiconaren & escucharán & $\begin{array}{l}\text { nacojé } \\
\text { quicunaren }\end{array}$ & & \\
\hline
\end{tabular}




\begin{tabular}{|l|l|}
\hline \multicolumn{2}{|c|}{ Ejemplos de algunas frases en pokomam } \\
\hline Veo muchas cosas & hen hincunilac maj pat \\
\hline Te veo & hen tincvuila yavua \\
\hline Tú me ves & het quinals yuben \\
\hline Él te ve & re quirela \\
\hline Los vemos & koj tikilata \\
\hline Nos ven & heta kahala \\
\hline Ellos me ven & retaque quiquela \\
\hline Entro en la casa & hen quinococo pan pat \\
\hline Llovió anoche & ejru anjam chakam \\
\hline La mujer busca a su hombre & re ixok eruric ru pabil \\
\hline El sombrero está tejido & re panes anojché \\
\hline Sombrerero & re enancvue panes \\
\hline Como tortillas & hen nukux cruiic \\
\hline No como nada & hen kuchuku nukux \\
\hline Tomo agua & hen hinkuquej ja \\
\hline Muelo el maÍz & hen nuqueej ixim \\
\hline Hablo contigo & hen nanukutaj havuchpech \\
\hline Caigo en el piso & hen quinquejua pan acal \\
\hline Salgo de casa & hen quinelerajè pan pat \\
\hline Él entra en la casa de su padre & re enococo chiru pat rutat \\
\hline ¿Hay huevos en venta? & vuile moloj rehen kalok \\
\hline No hay nadie & nijam \\
\hline
\end{tabular}




\section{Notas}

* Publicación original: 1907, XV Congrès International des Americanistes (1906), Vol. 2: 423-465, Québec, Canadá. Traducción del alemán de Axel M. Köhler, revisión de Víctor M. Esponda J.

${ }^{1}$ Zur Ethnographie der Republik Guatemala, Zürich: Orell, Fuessli \& Co., 1884 [Etnografía de Guatemala, 1958. Seminario de Integración Social Guatemalteca. Editorial del Ministerio de Educación Pública, Guatemala, Centroamérica. Traducción de Antonio Goubaud Carrera. Nota de los Editores, en adelante N. de los E.].

${ }^{2}$ Ms. de la Biblioteca de Guatemala, copiado (y publicado) en Madrid, 1893.

${ }^{3}$ Incidents of Travels in Central America, Chiapas and Yucatan, Nueva York 1841[Existe una versión estractada en castellano de esta obra: Incidentes de viaje en Chiapas, Gobierno del estado de Chiapas, 1989, Miguel Ángel Porrúa, librero-editor, traducción de Juan C. Lemus, N. de los E.].

${ }^{4}$ Das nördliche Mittelamerika, Braunschweig [El norte de Mesoamerica, Brunswig], 1897, pp. 244, 348, 383, y 408 ss.; y los textos y mapas etnográficos en Petermann's Mitteilungen [Los Comunicados de Petermann] 1893, № 1 (Guatemala); 1895, No 8 (Südostmexiko und Britisch Honduras) [El Sureste de México y las Honduras Británicas], y 1901, № 2 (Südliches Mittelamerika) [El Sur de Mesoamerica].

${ }^{5}$ En la traducción de A. von Trautwein, Berlín, Nueva York, Londres, 1873.

${ }^{6}$ Arte y vocabulario en lengua choltí, Ms. de la colección Berendt, citado por Stoll (op. cit., p. 89).

${ }^{7}$ Historia de la Conquista de la provincia de el Itrá, reducción y progresos de la de el Lacandón, y otras naciones de Indios bárbaros, Madrid 1701.

${ }^{8}$ Estoy utilizando la ortografía española con las modificaciones propuestas por Stoll (op. cit., p. 39). Los frailes españoles introdujeron "tz" para el fonema "sch" en alemán [es decir, en el alfabeto fonético internacional: " "’ = "x" en la ortografía mexicana. N.de los E.]

${ }^{9}$ Hice un pequeño registro de datos lingüísticos en San Luis así como informes sobre la lengua en San Antonio. En comparación con el maya puro una de las grandes diferencias es un cambio fonético frecuente de "a" en "u": por ejemplo, mazorca: nul (en maya: na); totoposte [*]: sucpet (en maya: sacpet); carne: buk (en maya: bak); sabana: chuk'an (en maya: chak'an); mano: k'ul (en maya: k'al); cuatro: cumbel (en maya: can); seis: vukbel (en maya: vak); rojo: chuk (en maya: chak); blanco: suk (en maya: sak); amarillo: k'un (en maya: k'an); noche: $a k^{\prime} u$ (en maya: $\left.a k^{\prime} a b\right)$. En tales palabras encontramos cierta semejanza fonética con el chontal (por ejemplo, noche: ak'öb), porque en el chontal se ha dado un cambio fonético semejante. A veces se da también un cambio fonético de "l" en " $r$ ": mañana se traduce en San Luis como samar, en maya es samal. Igualmente existe un cambio fonético de " $\mathrm{t}$ " en "ch", por ejemplo, tu: inchech (en maya: tech); ustedes: inchex (en maya: teex). Además de las diferencias frecuentes ya mencionadas existen también otras en comparación con el maya puro: por ejemplo, peine: xeilch (en maya: xalche); estrella: xalab (en maya: eek); pequeño: tritzip (en maya: chanchan); siete: vukubel (en maya: vuk); nosotros: inoon (en maya: toon; chontal y chortí: noon). Pero en general las diferencias resultan ser tan mínimas que se pueden clasificar las lenguas de San Luis y de San Antonio como meros dialectos del maya. Cabe mencionar que en los tiempos de mi visita, es decir en 1891, la mayoría de los habitantes hablantes del cholchí de San Luis ya se habían mudado a San Antonio en las Honduras Británicas, mientras que San Luis fue colonizado por indígenas kekchí. ¡Así de rápido se pueden dar importantes cambios en las fronteras lingüísticas!

[*] Una variedad de totopo o tostada. [N. de los E.].

10 "Account of the Province of Verapaz, in Guatemala, and of the Indian Settlements or Pueblos established therein", Journal of the Royal Geographical Society, Londres, Vol XI, p. 14.

${ }^{11}$ Evidentemente una errata, debe decir Xucaneb.

* La parcialidad de Santo Tomás Apóstol es tan antigua como la Conquista misma, y fue poblada por indígenas lacandones que vivían al Norte de Cobán. De manera similar, Santo Domingo de Cobán fue establecido con indígenas que capturaban en las montañas de Chichén y Xucamel. Las cuatro parcialidades de San Pedro Carcha fueron pobladas por indígenas de los alrededores inmediatos. Habitualmente los indios de San Pedro y Cobán todavía van a recolectar productos de los lugares de aquella tierra que desde los tiempos antiguos pertenecía a sus respectivos antepasados [N. de los E.].

${ }^{12}$ Publicado en las Memorias del XIV Congreso de Americanistas en Stuttgart, pp. 373-381 y pp. 384-397. 
${ }^{13}$ Una localidad en el norte de Alta Verapaz que todavía es conocida hasta nuestros días. [Esta nota estaba ubicada en un lugar que no correspondía a la lógica del texto. N. de los E.]

${ }^{14}$ Los lacandones tenían en posesión una parte del botín que tomaron de los caídos (Villagutierre, p. 97). Poco después de la guerra, una parte de los lacandones, a saber, los habitantes de Topiltepeque, se asentaron en Verapaz bajo la protección de los dominicos, otra parte de ellos (los de Puchutla) estaban en negociaciones al respecto (Villagutierre, p. 78 s.), pero en 1564 se asentaron en Ocosingo, Chiapas, con Fray Pedro Lorencio (Remesal, 10, 17; 1640).

${ }^{15}$ Ver op. cit., p. 51.

${ }^{16}$ K. Sapper, Das nördliche Mittelamerika, Braunschweig 1897, pp. 334-353. Si los topónimos no nos apoyan en este sentido, hay otra indicación en favor de un poblado chol en aquellos tiempos en las zonas norteñas mencionadas, y es la siguiente: los indígenas kekchí que aparentemente subieron desde el sur se apropiaron de denominaciones claramente choles para varios de los animales antes no conocidos por ellos, por ejemplo: chak mut = "pájaro rojo", hocofaisán; chak ti $=$ "boca roja", un pez que existe en la región del río Usumacinta; ajau chan = "serpiente real", una especie de boa (cf. El norte de Mesoamerica, p. 397). Desgraciadamente estos términos no nos aportan ninguna certeza respecto al hablado de los lacandones y acalanes, dado que pueden ser préstamos de los choles del noreste.

${ }^{17}$ Die alten Ansiedlungen con Chacula I [Los antiguos asentamientos de Chaculá I], Berlín 1901, p. 11. Seler nos informa con bastante amplitud la historia de los lacandones en los siglos XVI Y XVII, por ello no lo hago yo aquí (op. cit.,pp. 5-13). Desgraciadamente, no hemos podido acertar la ubicación de las localidades particulares excepto la de Villa de Nuestra Señora de los Dolores cuya ubicación se puede definir relativamente bien a través de la siguiente indicación de distancia que tenemos: se encontraba a 32 leguas de la desembocadura del río Lacantún al río Usumacinta. Ciertamente no muy lejos de allá estaban ubicados también los asentamientos principales de los lacandones que se encontraban en una isla en medio de un lago, además las aldeas de Topiltepeque y Puchutla, así como las de Mop y Peta. Sin embargo, los conocimientos topográficos y arqueológicos de esta región son demasiado escasos para ubicar dichas localidades. Lo que es cierto, es que Peta, la localidad arriba mencionada, no es idéntica con el lago Pet Ha, el cual está situado mucho más al norte. Dicho lago, en cuyos alrededores viven ahora lacandones maya-hablantes, lo visité en 1894, Teobert Maler lo hizo en 1898, y más tarde Alfred Tozzer.

En Tenosique, donde una cantidad de lacandones buscaba refugio a finales del siglo XVII, algunas familias todavía hablaban chol a mediados del siglo XIX, pero no sabemos si estas familias son descendientes de dichos refugiados o inmigrantes choles de tiempos más recientes. Cuando yo visité Tenosique en 1896, ya no se hablaba chol allí.

${ }^{18}$ Villagutierre y Sotomayor (op. cit., p. 161).

Pero parece resultar del Ms. de la Historia de Ximénez que estos números se refirieron únicamente a los choles asentados al norte del lago Ysabal. Al respecto, Ximénez nos dice lo siguiente: "La nación chol en tiempo de su gentilidad estuvo poblada en todas las tierras, que hoy comprende Chiquimula de la Sierra, Esquipulas, Cazaguastlán y todas aquellas montañas, que están de la otra parte del Golfo y río que se llama del Castillo, hacia la provincia de la Verapaz y más hacia lo que se llama el Petén, pero éstos fueron pocos respecto a los muchos que comprendían las tierras dichas de Chiquimula etc., de cuya nación chol se fundaron todos estos curatos, aunque algunos de ellos muy deteriorados al día de hoy por los muchos indios que consumió la guerra que fue muy sangrienta. Toda esta nación chol componía un reino de mucha fuerza, que es el que llamaron de Copan, como lo demuestran las grandes ruinas de sus edificios que no se ven tales en todas aquestas provincias". [Existe una versión publicada de este Ms.: Ximenez, F. 1930/1931. Historia de la Provincia de San Vicente de Chiapasy Guatemala. 3 Volumenes, Guatemala. N. de los E.]

${ }^{19}$ Villagutierre, p. 163.

${ }^{20}$ Villagutierre, p. 174.

${ }^{21}$ Villagutierre, p. $188 \mathrm{~s}$.

${ }^{22}$ Villagutierre, p. 277 s.

${ }^{23}$ Villagutierre y Sotomayor, pp. 384-387.

${ }^{24}$ Villagutierre y Sotomayor, p. 153. Los indios kekchí en la selva todavía siguen venerando de la misma manera su Tzultacca pagano junto con el Dios cristiano. Véase Sapper, Das nördliche Mittelamerika [El norte de Mesoamerica], pp. 267 ss.

${ }^{25}$ Entre tales apellidos se encuentran: Cucul, "techo" (Villagutierre, p. 175) y Cabnal (Ibid., p. 309).

La mayoría de los apellidos en Alta Verapaz, parece ser kekchí: en gran medida son nombres de animales y plantas o son tomados de objetos hechos de la flora o fauna, por ejemplo, Chub = avispa; Chen = mosquito; Hor = chaquiste; $T_{\text {z } i}=$ perro; $M_{o}=$ guacamaya (ara); Pap = una especie de ave; 
Choj $=$ otra especie de ave; $C o c=$ tortuga; $B a=$ tuza $($ Geomys hispidus); $C u c=$ ardilla; $C h o c o j=$ especie de mariposa; $K^{\prime} a k=$ pulga; $P$ ou = pavo (real) salvaje; $B a c=$ hueso, y otros; así que Che = árbol; Quix = espina (espiga); May = tabaco; $\mathrm{O}_{0}=$ aguacate (Persea gratissima); Tul= plátano; $C a c a o=$ cacao; $I_{c}=$ chile (Capsicum annaum); Ixim = maíz; $X e=$ raíz; $A x=$ mala hierba de grandes hojas; Koy y Siguic = otras especies de plantas; K'aal = especie de árbol; Ical, Yixcal y Yaxcal = milpa; Guim = pasto, paja (zacate?); también se encuentran denominaciones de colores en los apellidos (Yax = verde; Can = amarillo) u objetos naturales $($ Trul $=$ montaña; $P e c=$ piedra; $K u=$ estalactita; $M a c s=$ telpetate o piedra pomes, una piedra suave), igual que objetos trabajados (Cus = bola; Pop = estera, Xol $=$ flauta; Tun = tambor, teponastle).

Después de la conquista, tales apellidos parecen ser muy comunes. En Chamá, sin embargo, a donde bastante gente del barrio San Marcos de Cobán emigraron desde sus lugares de origen, existe la costumbre que un hombre toma como apodo el nombre del primer objeto que le llama la atención al salir de la casa después de la noche de boda. Este apodo lo utilizan únicamente los compañeros de su etnia. Durante mi estancia en Chamá en 1891, hice una lista de los nombres y apodos de los indios de allí, de los cuales daré unos ejemplos. ${ }^{26}$ K. Sapper, Die Alta Verapaz und ihre Bewohner [Alta Verapaz, y sus habitantes], Ausland [Extranjero] 1891, № 51 y 52. Ver también Petermann's Mitteilungen [Los Comunicados de Petermann] 1893, p. 7 s.; Ibid., 1895, p. 180 s.; Die Alta Verapaz. [Alta Verapas], Hamburgo 1902.

${ }^{27}$ A.M. Tozzer, A comparative study of the Maya and the Lacandones, Arqueological Institute of America, Nueva York, 1907, pp. 3 y 40 ss. [Existe una versión en castellano de esta obra: Tozzer, A.M. 1982. Mayas y lacandones. Un estudio comparativo. México: Instituto Nacional Indigenista, Colección Clásicos de la Antropología, núm. 13, N. de los E.]

* No se incluyó este mapa en la publicación original. N. de E. * "sch" corresponde al fonema " $"$ " = " $x$ " en la ortografía mexicana. Cabe señalar que las vocales con diéresis utilizadas por Sapper parecen corresponder al alfabeto alemán, son los llamados «Umlaute»: 'ä', 'ö’ y 'ü’ que se pronuncian como vocales situadas entre las vocales correspondientes sin diéresis, es decir, 'a', 'o' y 'u' y el vocal 'e'. [N. de los E.]

${ }^{28}$ Una manera apresurada de hablar en El Obraje; traducido correctamente sería así: duermo en mi casa $=$ nen inconayan ta nicvotot.

${ }^{29} \mathrm{La}$ inclusión de 'c' antes de 'vu' es muy común en las lenguas del grupo pokom.

30 A menudo escuché claramente «cansa» o «cansam»; aparentemente se trata de una pronunciación apresurada que es bastante común. 